\title{
High-Resolution Bent-Crystal Spectrometer for the Ultra-Soft X-ray Region
}

\author{
P. Beiersdorfer ${ }^{\dagger}$, S. von Goeler, M. Bitter, \\ K. W. Hill, R. A. Hulse, \\ Princeton University, Plasma Physics Laboratory, Princeton, \\ New Jersey 08543, USA \\ R. S. Walling \\ University of California, Lawrence Livermore National \\ Laboratory, Livermore, CA 94550, USA
}

\begin{abstract}
A multichannel vacuum Bragg-crystal spectrometer has been developed for high-resolution measurements of the line emission from tokamak plasmas in the wavelength region between 4 and $25 \AA$. The spectrometer employs a bent crystal in Johann geometry and a microchannel-plate intensified photodiode array. The instrument is capable of messuring high-resolution spectra $(\lambda / \Delta \lambda \approx 3000)$ with fast time resolution (4 msec per spectrum) and good spatial resolution $(3 \mathrm{~cm})$. The spectral bandwidth is $\Delta \lambda / \lambda_{0}=8 \AA$. A simple tilt mechanism allows access to different wavelength intervals. In order to illustrate the utility of the new spectrometer, time- and space-resolved measurements of the $n=3$ to $n=2$ spectrum of selenium from the Princeton Large Torus tokamak plesmas are presented. The data are uned to determine the plasma transport parameters and to infer the radial distribution of fluorinelike, neonlike, and sodiumlike ions of selenium in the plama. The new ultra-soft $x$-ray spectrometer hes thus enabled us to demonstrate the utility of high-resolution L-shell spectroscopy of neonlike ions as a fusion diagnontic.
\end{abstract}

\footnotetext{
†Present Addreas: Univeraity of California, Lewnence Livermore National Leboralory, Livermore, CA, 94550
}

\section{MASTER}




\section{Introduction}

High-resolution spectroscopic observations on tokamaks are routinely used to determine the concentrations and transport of impurity ions in the plasma as well as to measure bulk plasma motion and to monitor the efficiency of ion heating. ${ }^{1-3}$ Such observations also have provided valuable data for comparison with basic atomic physics calculations and models.

Tokamak spectroscopy has been performed with high resolution primarily in the soft $x$-ray region below about $4 \AA$ and in the extreme ultra violet (XUV) region above about $25 \AA$. High-resolution instruments employed in the soft $x$-ray region typically consist of a bent-crystal spectrometer and a singlewire or multi-wire gas proportional counter. ${ }^{4-8}$ Due to the need for windows to separate the counter gas from the tokamak vacuum these instruments have low efficiencies for $x$ rays with wavelengths above about 4 A. Multichannei grazing incidence spectrometers with microchannel plate-intensified photodiode readout have recently been developed for use in the XUV region. ${ }^{0,10}$ The spectral resolution and the grating reflectivity of such instruments decrease considerably at the shorter wavelengths below about $25 \AA$. As a result of the instrumental limitations the intermediate ultra-soft $x$-ray (USX) region from 4 to $25 \mathrm{~A}$ has been surveyed mainly with low resolution.

In this paper we describe a new multichannel spectrometer with a microchannel plate-intensified photodiode array designed to study the ultrasoft $x$-ray region with high spectral rewolution and fast time response. The new instrument iucorporates design features from the soft $x$-ray and XUV spectrometers. Like an $x$-ray spectrometer it uses a bent crystal in Johann geometry ${ }^{11}$ for mavelength dispersion. Like a UV spectrometer it employs a microchannel plate-intensified photodiode assembly for position-sensitive detection and operates in vecuo. The new USX spectrometer allows the recording of a high resolution spectrum $(\lambda / \Delta \lambda \approx 3000)$ every 4 maec. It is equipped with a shot-to-shot poloidal scanning capability across the plasma minor cross section of the tobamak for measuring radial profiles of the spectral line emiscion. The spatial resolution is $3 \mathrm{~cm}$ at the center of the planma which corresponds to $7.5 \%$ of the plasm radius of the Princeton Large Torus 
(PLT) tokamak. In a single setting the instrument covers a spectral range of $\Delta \lambda=1.2 \AA$ at a nominal wavelength of $\lambda_{0}=8 \AA$. A simple tilt mechanism has been devised to position the detector to difierent Bragg angles and thus to access neighboring spectral regions.

To illustrate the versatility of the new USX spectrometer we have performed detailed measurements of the $n=3$ to $n=2$ spectrum of selenium as a function of time and of plasma radius on the PLT tokamak. For this purpose the spectrometer has been used to observe the wavelength region $7.2-9.0 \AA$ which contains the $3 d \rightarrow 2 p$ resonance transitions of neonlike selenium as well as the resonance transitions of the neighboring fluorinelike and sodiumlike charge states. ${ }^{12}$ The data have been used to infer the impuritytransport parameters and to determine the radial charge-state distribution of selenium in the plasma. The measurements show that the spectral emission of neonlike ions provides an excellent source for plasma diagnostic purpoess, first, because the $x$-ray emission of neonlike ions is found to be very bright, and second, because neonlike ions extend over a large central and near-central volume of the plasma due to their closed-shell configuration. The new USX vacuum bent-crystal spectrometer has thus provided us with a novel way of studying fusion plasmas which complements the study of $\Delta n=0$ transitions performed with XUV and vacuum ultra violet (VUV) instruments and the study of $\Delta n=1$ transitions of hydrogenlike and heliumlike ions in the soft $x$-ray region.

The paper is organized as follows. In Section I we give a description of the general design of the new spectrometer. The detector assembly and readout are described in Section Il. A brief discussion of the crystal bending is given in Section III, and the results of testing and operating the opectrometer are presented in Section IV. In Section V we describe the simple tilt mecheniom used to position the detector to different Bragg angles on the Rowland circle. Finally, in Section VI, we present typical results from the transport studies on the PLT tolamak. 


\section{General Design Characteristics}

Since bent-crystal spectrometers provide superior throughput characteristics compared to non-focussing, flat-crystal spectrometers, the new USX spectrometer was built in the Johann geometry. "The focussing properties of the Johann geometry are shown in Fig. 1 . X rays witt wavelength $\lambda$, emanating from the shaded region, are reflected by the crystal according to Bragg's law.

$$
n \lambda=2 d_{n} \sin \theta .
$$

Here $d_{n}$ is the lattice spacing of the crystal, $n$ is the order of diffraction, and $\theta$ is the Bragg angle, i.e., the angle between the $x$ rays and the tangent to the crystal surface. For a crystal bent to a radius of curvature $R_{c}$, the reflected $x$ ray's of a certain energy are focussed on the Rowland circle of radius $R_{c} / 2$ tangent to the crystal. $\mathrm{X}$ rays with different energy come from different regions in the plasma and are focussed at difierent points along the Rowland circle.

A schematic of the new USX spectrometer is shown in Fig. 2. Since we are interested in measuring the spectrum of neonlike selenium, the nominal Bragg angle of the new spectrometer was set to $50^{\circ}$. With an ADP crystal cut along the 101 plane having a $2 d_{\infty}$-spacing of $10.648 A,{ }^{13}$ this angle corresponds to the center of the $3 \rightarrow 2$ spectrum of neonlike selenium at $\lambda=8.16 \AA$. The $100-\mathrm{mm} \times 20-\mathrm{mm} \times 0.5-\mathrm{mm}$ ADP crystal is held in a four-bar bending jig, ${ }^{4,14}$ which is mounted on a rotary feedthrough connected to a stepper motor. Interchangeable apertures are placed in front of the crystal which allow adjustment of the illuminated length of the crystal. The apertures are covered with an ultre thin, 900 A-thick aluminum foil. The foil attenuates UV and visible light and thus reduces the heat load on the crystal. A second foil, convisting of 10,000 A-thick parylene between two $3000 A$-thick layers of magnexium, is placed between the crystal and the detector. The foil is mounted on a frame bolder with pumping ducts. It is transparent to soft $x$-ray radiation, but acts to shield the detector from stray UV and visible radiation. The absorption characteristics of the combination of the aluminum and magnesium-parylene foils are shown in Fig. 3. The foil between the 
crystal and the detector also serves to mitigate pressure surges arising during plasma startup and from plasma disruptions by keeping surges from quickly reaching the detector. This is especially important because the spectrometer is directly connected to the PLT vacuum. The output from an ion gauge located near the detector is interfaced to a protective circuit which disables the high voltage on the detector, if the pressure exceeds $2.0 \times 10^{-6}$ torr. A pivot point near the PLT racuum vessel enables the spectrometer to move poloidally by $14^{\circ}$ above and $17^{\circ}$ below the horizontal midplane for radial profile measurements.

\section{Detector Assembly and Readout}

In the wavelength region below bout $4 A$ it is customary to use singlewire or multi-wire proportional counters for the detection of $x$ rays in Bragg crystal spectrometers on tokamaks. ${ }^{4.5 .7}$ Because such detectors are filled with a counter gas, it is necessary to use a window to separate the gas chamber from the tokamak vacuum. Due to the absorption of low energy photons by the vindow, proportional counters se inefficient for the detection of ultrasoft $x$-radiation in the wavelength range above about $4 \mathrm{~A}$. As a consequence, the new racuum spectrometer has been equipped with a position-sensitive microchannel plete-intensified photodiode array detector which can operate in vacuo.

The design of the microchannel plate-intensified photodiode array detector followed an arrangement used by Fonck et $a .^{0,15}$ in the detection of VUV radiation. A schematic of the detector employed in the new spectrometer is shown in Fig. 4. The design of Fonck et al. was modiffed to reflect the reduced senvitivity of microchannel plates at higher photon energiea. For example, instead of uring a single microchannel plate the present detector employs two microchennel plates in a Chevron configuration. ${ }^{16}$ The $15-\mathrm{mm} \times 100-\mathrm{mm}$ plates are butt-mounted for higher resolution. The front plate was coated with approximately $3000 \AA$ of CsI, which has an electron emission rate per soft $x$ ray about three times higher ${ }^{17}$ than that of the $\mathrm{CuI}$ coating used by Fonck et al." The secondary electrons are proximity focused onto the fiber 
optic surface of a $7.5 \mathrm{~cm}$-long optical extender. The surface is coated with about $8 \mu \mathrm{m}$ of P-20 phosphor and backed with a thin film of SnI to prevent buildup of electrostatic charges. The time response of P-20 is approximately 2 msec, as measured by Fonck et al. The separation between the phosphor screen and the back of the microchannel plate is $0.5 \mathrm{~mm}$. The optical extender is coupled via a fiber optical face plate to two optical tapers. The face plate provides the vacuum-air interface. The tapers image the $10 \mathrm{~cm}$ long light output from the phospor via two light-fiber bundles onto two 2.5 cm-long, self-scanning RC1024SF Reticon photodiode arrays of :024 pixels each. ${ }^{18}$

Each photodiode array is read with the help of an RC1024S-3 Reticon evaluation circuit, ${ }^{10}$ as shown in Fig. 5. The time between the readout of two consecutive pixels is $4 \mu \mathrm{sec}$ and is determined by the clock rate of 250 $\mathbf{k H z}$. Thus the time to read the entire array is approximately $4.1 \mathrm{msec}$. Each readout is started by a start-of-scan pulse and ended by an end-of-scan pulse. The evaluation circuit allows for the insertion of delay times of up to 64 msec between consecutive readouts and thus for the adjustment of the integration time. The minimum delay time is $0.26 \mathrm{msec}$. The clock pulse from the evaluation board is used to trigger a Lecroy 8210 10-bit ADC which digitizes the video signal as well as the start-of-scan pulse. A logic circuit suppresses clock pulses between an end-of-scan and a start-of-scan pulse, and thus prevents the digitization of an empty video line. The lecroy 8210 digitizer operates in a post-trigger sampling mode, i.e., digitization begins following a trigger signal which in the present setup is provided by the PLT master timer. This arrangement causes the start of the digitization to be uncertain in those cases where the trigger signal from the PLT timer happens to fall in the time interval between two consecutive readouts of the Reticon array. Three Lecroy 8800 memory modules are used to store 96k of data for each Reticon array. This corresponds to approximately 48 spectre and 48 ascocinted start-of-scan pulses for each array per shot. After the tolcamak pulse the data are read out to a Vax 11/785 data acquisition computer for permanent archiving and analysis.

The detector is mounted on a bellows assembly as shown in Fig. 2. This 
allows moving the detector past the gate valve onto the Rowland circle when making measurements; the detector can be retracted and thus kept under vacuum while changes to the spectrometer, such as an exchange of crystals, are made.

The size of the instrument is determined by the size of the detector, the desired resolving power, and the spectral rarige we wish to observe in a given setting. The dominant electric dipole transitions $3 d, 3 s \rightarrow 2 p$ of neonlike selenium lie in the wavelength interval 7.6-8.7 $\AA$. This corresponds to Bragg angles between $45^{\circ}$ and $55^{\circ}$, or $\Delta \theta=10^{\circ}$. Thus, we require that the detector subtend an angular range of $\Delta \theta=10^{\circ}$ centered at $\theta=50^{\circ}$. Since the active area of the detector is $10 \mathrm{~cm}$ long, it follows that the diameter of the Rowland circle has to equal $R_{c} \approx 57 \mathrm{~cm}$.

A consequence of the small Rowland circie is that the spectrometer has to be placed close to the tokamak vessel in order to reduce the amount of vignetting due to the vacuum port. As a result, we have located the spectrometer near the perimeter of the toroidal field coil, as shown in Fig. 2. This represents the closest distance possible without creating interference problems with the poloidal motion of the spectrometer. Unfortunately, this distance was not close enough to prevent all vignetting by the edges of the vacuum port.

Having fixed the size of the spectrometer we can make an estimate of the dominant focussing defects and thus of the anticipated resolving power of the instrument. The resolving power $\lambda / \Delta \lambda$ of the spectrometer at a $\mathrm{Brags}$ angle $\theta$ can be found from Eq. (1) and is given by

$$
\lambda / \Delta \lambda=\frac{1}{\Delta \theta} \tan \theta .
$$

The angular resolution $\Delta \theta$ of the detector is determined by the spatial resolution of its components and its distance from the crystal. The pore size of each microchannel plate is $25 \mu \mathrm{m}$. At a radius $R_{c}=57 \mathrm{~cm}$, the angular resolution of the Chevron corresponds thus to a resolving power of about 14,000 . The Reticon has a pixel spacing of $25 \mu \mathrm{m}$; however, each pixel views a 50- $\mu \mathrm{m}$ wide area on the phosphor screen so that the resolving power of the Reticon equals about 7,000 ; similarly, the resolving power of the light 
fiber bundle ( $7 \mu \mathrm{m}$-diameter fibers) corresponds to 40,000 . For comparison, the Doppler-broadened linewidth of selenium corresponding to a typical ion temperature of $1 \mathrm{keV}$ is about 4,000 . Hence the resolving power of the individual detector components is more than adequate for our measurements. The same is true for the intrinsic resolving power of the crystal which equals about $8000 .^{13}$

The resolving power of the spectrometer is not only determined by the resolving power of its components, but it is also affected by a variety of focussing defects. First, the Johann geometry is not exactly focussing. ${ }^{11}$ Instead it has an associated focussing erior which depends on the illuminated length $l_{c}$ of the crystal and is given by ${ }^{11}$

$$
\Delta \theta_{1}=\frac{l_{c}^{2}}{8 R_{c}^{2} \tan \theta} .
$$

Another focussing defect results from the height of the crystal $h$. This socalled vertical error is given by ${ }^{19}$

$$
\Delta \theta_{2}=\frac{h^{2}}{8 R_{c}^{2} \sin \theta \cos \theta} .
$$

A ihird error is due to the fact that the detector is straight and thus tangent to the Rowland circle at best only in one spot. Assuming that it is tangent in the center, the worst focussing defect occurs st the edges of the detector and can be obtained from geometrical optics as

$$
\checkmark J_{3}=\frac{l_{c} l_{d}^{2}}{8 R_{\tau}^{3} \sin ^{2} \theta},
$$

where $l_{d}$ is the length of the detector. Moreover, the error due to misaligning the center of the detector a distance $\Delta d$ from the Rowland circle results in an error

$$
\Delta \theta_{4}=\frac{\Delta d l_{c}}{R_{c}^{2} \sin \theta} .
$$

Since all four errors, Eqs. (3)-(6), depend on the illuminated length and width of the crystal, they can be made arbitrarily small by reducing the illuminated area of the crystal. We are only limited by the spectral intensity 
as well as by the resolving power of the ADP crystal, which in Ref. 13 is given to be $\lambda / \Delta \lambda=8200$. The moveable apertures in front of the crystal allow a variation of the illuminated length of the crystal from $1.3 \mathrm{~cm}$ to 2.9 $\mathrm{cm}$. The shortest length of $1.3 \mathrm{~cm}$ was found to be by far sufficient to record bright spectra even at the fastest possible time resolution of the spectrometer $(\Delta t=4 \mathrm{msec})$. For $l_{c}=1.3 \mathrm{~cm}, l_{i}=10 \mathrm{~cm}, \theta=50^{\circ}, h=2 \mathrm{~cm}, \Delta d=0.2$ $\mathrm{cm}$, and $R_{c}=57 \mathrm{~cm}$ the dominant of the four errors given in Eqs. (3)-(6) is the vertical error. The value of $\lambda / \Delta \lambda$ corresponding to this error is 3800 , while $\lambda / \Delta \lambda$ corresponding to the sum of all four errors is about 3500 . As noted earlier this value is approximately equal to the Doppler width of most lines at $1 \mathrm{keV}$ ion temperatures typical in PLT.

\section{Crystal Bending}

The $100-\mathrm{mm} \times 20-\mathrm{mm} \times 0.5-\mathrm{mm}$ ADP crystal was bent to a cylindrical form by using a four bar bending jigi,14 shown in Fig. 6. The radius of curvature of the crystal is adjusted by means of the fine-thread screws which hold the top rollers. Four copper-beryllium springs, one on each side of each top roller, firmly press the rollers onto the adjustment screws and counteract the weight of the rollers. This arrangement was necessary because the weight of the rollers alone was sufficient to bend the crystal to a radius of curvature less than the radius desired.

The quality of the curvature of the crystal was checked optically by using reflection of a divergent laser bearn from the front surface of the crystal. The sharpness of the focus of the reflected beam was determined using a Reticoia photodiode array. Typical Reticon traces are shown in Fig. 7 for five different distances from the crystal. The illuminated length of the crystal in this case was $2 \mathrm{~cm}$. We note that as the Reticon is moved away from the focal point the image becomes inureasingly more structured. In particular, the single focal line in Fig. 7 (c) splits into two lines which separate as the distance is increased, as seen in Figs. 7(a) and (b). By covering the left or right half of the crystal, we find that one of the two lines emanates from the right, the other from the left half of the crystal. Decressing the distance leads to 
the emergence of wings to either side of the central line which spread and grow as the distance is further increased, as seen in Figs. $7(d)$ and (e). As the distance is increased or decreased further, each line bifurcates into additional lines. If we increase the illuminated length of the crystal to $5.0 \mathrm{~cm}, F i g .7(\mathrm{f})$, additional structure is visible. The multiple structure seems to indicate that the crystal does not bend uniformly, possibly as a result of variations in the thickness of the crystal. ${ }^{6}$

The sharpness of the focus as a function of distance is shown in Fig. 8. Here the focal width is defined as the full width at half-maximum of the measured peak. In the case where two or more distinct lines were visible, the width is defined so as to enclose the two outermost lines on each side at half maximum. The focal width was determined also for a second ADP crystal with a thickness of only $0.25 \mathrm{~mm}$. This crystal was polished on one side only. As seen in Fig. 8, the thicker crystal provided superior focussing characteristics. Its sharpest focus was about four times better than the focus of the thinner crystal. More importantly, the thicker crystal could be focussed in a much shorter time than the thinner crystal, and it maintained its radius of curvature throughout the length of this investigation, while the thinner crystal had to be readjusted periodically. In addition, the line focus of the thinner crystal was not uniform along its length. Using Eq. (6) we can calculate a simple estimate of the focal width of the crystal based on the distance of the Reticon from the focal point. This estimate is plotted as a solid line in Fig. 8. It roughly agrees with the observed defocussing although the simple estimate does not account for the initernal structure exhibited by the observed focus. Figure 8 shows that a resolution $\lambda / \Delta \lambda \geq 5000$ can b: maintained in a region of $\pm 0.5 \mathrm{~cm}$ surrounding the location of best focus. Exact alignment of the detector on the Rowland circle is, therefore, not necessary.

\section{Testing and Operation}

Using \& PHI Model 04-151 commercial x-ray source ${ }^{20}$ with an aluminum target, we heve measured the signal level of the detector as a function of 
the voltage on the phosphor screen, $V_{P H}$, and as a function of the voltage across the microchannel plates, $V_{M C P}$. The results are shown in Fig. 9 . As is expected, the signal gain depends more strongly on the microchannel plate voltage, and is less sensitive to the accelerating potential on the phosphor. On PLT we have usually operated the detector with voltages $V_{M C P}=-1300 \mathrm{~V}$ and $V_{P H}=2500 \mathrm{~V}$. Although the detector had been tested at up to $V_{P H}=$ $5000 \mathrm{~V}$ in the laboratory, the detector could not be operated at such high voltages without the risk of arcing after the spectrometer was installed on PLT, because of a higher base pressure in the system.

An indication of the resolving power of the spectrometer is given by line widths measured on PLT. A typical spectrum obtained on PIT is shown in Fig. 10. The observed width of the line labelled Ly- $\zeta$ is $\lambda / \Delta \lambda \approx 2500$ and corresponds to some of the narrowest line widths measured with the new instrument. This line was recorded near the center of the detector. For comparison, the line labelled Ly- $\gamma$ was recorded close to the edge of the detector, and its width is only $\lambda / \Delta \lambda \approx 1600$. Assuming a Gaussian instrumental response function, the width of a measured line profile is given by

$$
\left(\frac{\Delta \lambda}{\lambda}-\right)_{\text {meanured }}=\sqrt{\left(\frac{\Delta \lambda}{\lambda}\right)_{D_{\text {oppler }}^{2}}^{2}+\left(\frac{\Delta \lambda}{\lambda}\right)_{\text {instrument }}^{2}} .
$$

Using this assumption and noting that $\lambda / \Delta \lambda_{\text {Dopper }} \approx 3500$ for sodium at the measured ion temperature of $0.5 \mathrm{keV}$, we find that the resolving power of the instrument at the center of the detector approximately equals the Doppler width of the line Ly- $\zeta$, namely $\lambda / \Delta \lambda \approx 3500$. This value is in agreement with the value we expect from the size of the focussing defects given by Eqs. (3)-(6) and discussed earlier.

The resolving power of the new vacuum crystal spectrometer was found to deteriorate with increasing microchannel plate voltage. Results of a scan of $V_{\text {MCP }}$ between $1100-1500 \mathrm{~V}$ are shown in Fig. 11. The scan was taken on PLT by measuring the width of several different lines indicated by the different symbols. $V_{P H}$ was kept fixed. Over the range of $V_{M C P}$ investigated the line width broadened by $30 \%$. In a similar fashion we have measured the line width as a function of $V_{P H}$, as shown in Fig. 12. The line width was 
found to be fairly insensitive to changes in $V_{P H}$. This result does not agree with the results obtained by Fonck et al. ${ }^{8}$ They observed an improvement of the resolution proportional to $\sqrt{V_{P H}}$.

Keeping both $V_{M C P}$ and $V_{P H}$ fixed, we notice an increase of the line width as the intensity of the line increases. The results are shown in Fig. 13. As the intensity quadruples, the line broadens by $30 \%$. The behavior can also be seen in the spectrum in Fig. 14. Here the FWHM of the strongest line is about $50 \%$ larger than that of the smaller lines. Fonck et al..$^{9,15}$ have studied the resolving power of intensified photodiode arrays extensively. However, they did not observe such a broadening of the line width with $V_{M C P}$ or line intensity. These researchers have used single microchannel plates in their detector, while we have used two. The secondary electron gain in a Chevror is about two orders of magnitude larger than that of a single microchatnel plate. ${ }^{21}$ As a result it could be possible that space charge effects in the gap between the back of the Chevron and the phosphor screen play a role in determining the resolution of our detector. This, however, does not explain the lack of noticeable improvement in the focussing properties at high voltages on the phosphor. The lack of improved line widths with $V_{P H}$, on the other hand, can be explained by saturation of the Chevron. However, we have found no supporting evidence for this possibility.

As mentioned earlier, the resolution of the spectrometer can be improved by reducing the illuminated area of the crystal. All data in this study were recorded using $l_{c}=1.3 \mathrm{~cm}$. The signal strength is sufficient to allow a reduction of $l_{c}$ by a factor of two or more. Concurrently, the illuminated width of the crystal can be narrowed in order to reduce the vertical error. This reduction would increase the resolution of the spectrometer well beyond the line width due to Doppler broadening.

The noise level in the readout of a 4 msec-long dark scan is about 23 counts on a 10-bit digitizer. The noise level is about twice as high in a 4 msec-long scan taken on PLT. Part of the increase is due to fluctuations in the radiation from the plasma itself; the remainder is due to pickup of electrical noise generated during a discharge. The best ratio of $x$-ray counts to noise is thus about 200:1 for a strong $x$-ray line. Adding several scans 
can improve this ratio considerably by reducing the statistical error. The noise leve] due to dark currents in the Reticon can be reduced by cooling the array, ${ }^{22}$ e.g., by using a Peltier thermoelectric cooler. However, no cooling has been used in the present setup.

Apart from random noise, Reticons exhibit fixed-pattern noise due to spatial variations in the transient signals coupled into the video line through stray capacitances in the clocks. This noise results in a systematic offset in every fourth pixel as shown in Fig. 15. The noise can be minimized by careful alignment of the Reticon Evaluation Board. Iowever, the effect changes in time, and such an alignment is impractical during operation on PLT. Instead, the offset of each of the four traces is found in a numerical algorithrn, and the offsets are removed during data analysis. The data of Fig. 14, for example, are the same as in Fig. 15 except that the data have been adjusted for the fixed-pattern offset. Spatially fixed noise patterns of arbitrary periodicities can also be eliminated by simply subtracting a dark scan from the data scan.

The effect of magnetic fields on the performance of the microchannel plates has been described in Ref. 23. Depending on the orientation of the field lines to the channel axes, a magnetic field can increase, diminish, or even completely inhibit gain in the microchannel plates. Furthermore, Fonck, who has used a microchannel plate-intensified photodiode array near the vacuum vessel of the PDX tokamak, has noted that the presence of magnetic fields (typically $10 \mathrm{kG}$ ) tends to stift the spectrum by a few pixels from the nofield case. ${ }^{24}$ We have minimized the effect of the toroidal magnetic field by placing the spectrometer near the outer edge of the toroidal field coil ( $c$. Fig. 2). This location is close to the point where the toroidal field reverses, and the detector sees less than $5 \%$ of the nominal field ( $30 \mathrm{kG}$ ) inside the torus. The detector may be more sensitive to the relatively stronger vertical magnetic field used to stabilize the plasma and to the stray field of the ohmic heating transformer. Shifts of the position of a given spectral line between 1 and 3 pixels have been observed on a shot-to-shot basis as well as between consecutive time groups in the same shot. The cause of these shifts, however, could not be determined, although the shifts may be relateo to changes in the vertical or ohmic heating magnetic fields. 
In order to determine the spatial variations in the intensity response of the detector, the detector was tested in the laboratory by sweeping it across the aluminum $\mathrm{Ka}$ line using the tilt mechanism between the detector and the crystal. The resulting traces recorded with the two Reticon arrays are shown in Fig. 16. The variations in the response appear to be due to nonuniformities in the thickness of the phosphor and due to the quality of the optical coupling between the light fibers and the Reticon. This method of testing the detector response does not account for variations which are functions of wavelength. However, the sensitivity of the detector as a function of photon energy is expected to vary only slightly, since the spread in wavelengths in the spectral range considered is relatively small. Moreover, the decrease in the transmission properties of the aluminum and magnesium-parylene foils for lower photon energies is compensated in part by an increase in the efficiency of the CsI photocathode. ${ }^{17}$

An in situ measurement of the spectrometer response as a function of wavelength was made on PLT using the bremsstrahlung continuum radiation produced in a low-electron temperature, xenon-prefill, discharge with runaway electrons. For this case the bremsstrahlung radiation completely dominated the much weaker line radiation in the spectra. The result obtained for the variation of the detector response with position reproduces the traces shown in Fig. 16 to within $10 \%$.

\section{Access to Neighboring Wavelength Regions}

Ideally, we would like to be able to make well-focussed measurements at any Bragg angle. If we want to change the alignment of the spectrometer from a Bragg angle $\theta_{0}$ to a new Bragg angle $\theta_{0}-\Delta$, as shown in Fig. 17, the crystal has to be rotated by an angle $\Delta$, since the sightline between the plasma and the center of the crystal remains fixed. Further, the detector arm has to be rotated by an angle $2 \Delta$, and the distance $D$ between the detector and the crystal has to be changed to satisfy the Johann condition, i.e.,

$$
D=R_{c} \sin \left(\theta_{0}-\Delta\right)
$$


We also have to rotate the detector, in order to keep it tangent to the Rowland circle. In the following, we describe a low-cost arrangement based on a simple tilting mechanism which allows alignment of the detector on the Rowland circle for Bragg angles close to the nominal Bragg angle.

As shown in Fig. 17 realignment of the spectrometer to a new Bragg angle $\theta_{0}-\Delta$ can be accomplished by tilting the detector by an angle $\beta$ at a pivot point P. From Fig. 17 we find that

$$
\theta_{1}=\Delta, \quad \theta_{2}=2 \Delta, \quad \beta=3 \Delta, \quad b=2 a \cos \Delta,
$$

where $b$ is the distance between the pivot point and the center of the detector, $a$ is the distance between the pivot point and the center of the crystal, and $\theta_{1}$ and $\theta_{2}$ are defined in the figure. For the untilted position, $\Delta=0$, so that

$$
b=2 a=2 R_{c} / 3
$$

determines the location of the pivot point $P$. In the tilted position we have to readjust the length of $a$ and $b$, in order to satisfy the constraint on $D$ given by Eq. (8). Hence,

$$
a=R_{c} \frac{\sin \left(\theta_{0}-\Delta\right)}{\cos 2 \Delta+2 \cos \Delta}
$$

and

$$
b=2 R_{c} \frac{\sin \left(\theta_{0}-\Delta\right) \cos \Delta}{\cos 2 \Delta+2 \cos \Delta} .
$$

In other words, if we turn the crystal to a new Bragg angle $\theta_{0}-\Delta$, we can keep the detector in focus and tangent to the Rowland circle, if we (i) choose a pivot point at a distance $a$ from the crystal and a distance $b$ from the detector, and (ii) tilt the detector by $\beta$.

Figure 18 shows a schematic of the tilt mechanism used to keep the detector in focus when changing the Bragg angle of the spectrometer. The lengths $a$ and $b$ are adjusted by nuts $\mathbf{A}$ and $\mathbf{B}$. The degree of tilting is determined by nuts $A$. In the present case this adjustment was carried out manually. All components, however, are suitable for remote control.

The range of Bragg angles accessible with this design is limited by the size of the bellows. The bellows should be wide enough to avoid obscuring part of 
the detector in a very tilted position, and they should be long enough in order to allow for a large tilt angle. If a tilt angle of $\beta= \pm 90^{\circ}$ could be achieved, the corresponding change in the Bragg angle would be $\Delta=\beta / 3= \pm 30^{\circ}$. In practice, however, values of $\beta$ are limited to a much smaller range, say $\beta$ $\leqslant \pm 45^{\circ}$, which results in a range of Bragg angles $\theta_{0}-15^{\circ} \leqslant \theta_{0} \leqslant \theta_{0}+15^{\circ}$, which is still quite respectable. In the present case the bellows used in the construction of the new instrument limited $\beta$ to $\pm 8^{\circ}$. As a result, we have been able to look at a wavelength range $7.2 \AA<\lambda<9.0 \AA$. This compares to a range of $7.5 \AA<\lambda<8.7 \AA$ without the bellows.

The spectrometer has also been used to measure spectra in second order. A spectrum of heliumlike argon obtained in second order with the vacuum crystal spectrometer is shown in Fig. 19. This technique again increases the accessible wavelength region. The spectrum also shows that the CsI-coated microchannel-plate photocathode is sensitive to photons with energies above $3 \mathrm{keV}$, although the sensitivity is reduced to that at lower photon energies.

\section{Transport Measurements}

To iliustrate the versatility of the new USX spectrometer and, in turn, to demonstrate the usefulness of $L$-shell spectroscopy for plasma diagnostics, we have made a detailed study of the $\Delta n=1$ resonance transitions of neonlike selenium and of associated satellite transitions. The data are used to infer the impurity transport parameters (diffision and convection coefficients for transport from the center of the plasma radially outward) and the radial charge-state distribution, i.e., the relative abundance of each charge state of an element as a function of minor radius.

The neonlike charge state dominates over a wide range oi eiectron temperature due to the closed-shell configuration of the neonlike ion, because the ionization potential of $n=2$ electrons in neonlike ions is much higher than that of $n=3$ electrons in sodiumlike, magnesiumlike, etc., ions. Thus observation of neonlike line emission allows the study of transport in a large volume of plasma in the central or near-central region. The neonlike x-ray emission is very bright so that we bave been able to make measurements 
with good spatial resolution (width of sightline at plasma center $\Delta x=3$ $\mathrm{cm}$ ) and good time resolution ( $\Delta t=4 \mathrm{msec}$ ). By contrast, it is not possible to make transport measurements of neonlike ions using UV spectroscopy, because neonlike ions radiate only very weakly in this spectral region.

Because selenium is not indigeneous to PLT plasmas, it was injected into the quasi-steady-state phase of the plasma by ablation of a thin film of selenium from a glass slide via a laser pulse. ${ }^{25,20}$ The time history of the neonlike resonance transition $\left(2 p_{1 / 2}^{5} 3 d_{3 / 2}\right)_{J=1} \rightarrow\left(2 p^{6}\right)_{J=0}$, labelled $3 C$ in Fig. 14, is shown in Fig. 20. The data are obtained by viewing the plasma along a central chord, i.e., a sightline passing through the center of the plasma.

In order to infer the transport parameters from spectroscopic observations, it is customary to model the time-dependent line emission with an appropriate charge-balance and ion-transport code. The procedures have been detailed in previous transport studies, ${ }^{27-30}$ and in the following we shall limit our discussion to a brief overview of the methods. The $3 \rightarrow 2$ line emission was modelled using the Princeton 1-D time-dependent multi-species impurity ion and transport code MIST described in Ref. 31. The code solves a set of coupled rate equations for the density $n_{q}$ of each ion species of charge $+q$ using the ionization and recombination rates of Post et al. ${ }^{\text {D2 }}$ Furthermore, the code models radial transport from diffusive and convective contributions:

$$
\Gamma_{q}=-D \frac{\partial n_{q}}{\partial r}+v_{r} n_{q} .
$$

Here $\Gamma_{q}$ is the radial Alux for charge state $+q_{i} D$ is the diffusion coefficient which, for the present study, is taken to be independent of radius; $v_{r}$ is the convective velocity. The convective velocity is parametrized by introducing a parameter $c_{v}$ and setting ${ }^{30}$

$$
v(r)=c_{v} D \frac{\partial \ln \left(n_{e}(r)\right)}{\partial r} .
$$

This parametrization ensures that the total impurity density profile $n_{\text {Inp }}=$ $\sum_{q} n_{q}(r)$ is proportional to the electron density profile, in particular $n_{I m p} \propto$ $\left[n_{e}\right]^{a_{1}}$, in the source/sink free region of the plasma. ${ }^{33}$ For the present study the electron density, $n_{e}$, along with the electron temperature, has been messured 
with Thomson scattering. Having determined the density of each ion species, the code then calculates the emissivity of a given spectral line based on excitation rates calculated by $\mathrm{C}$. Cerjan at Livermore using the code of Ref. 34 and on branching ratios of Chen. ${ }^{35}$ Results of the model calculations are shown in Fig. 21. The two transport parameters $D$ and $c_{v}$ have been varied systematically until the best fit to the data was achieved. The adopted values are $D \approx 5 \times 10^{3} \mathrm{~cm}^{2} / \mathrm{sec}$ and $c_{v} \approx 0$. The uncertainties in these values typically are large because the values are affected by uncertainties in the atomic rate coefficients and by uncertainties in the electron temperature profiles. The values are also affected by the details of modelling the socalled scrape-off region outside of the plasma limiter radius, as noted in Ref. 33. (For this study we have set the effective size of the scrape-off region equal to $2 \mathrm{~cm}$.) Furthermore, the possibility of re-entry of ions into the plasma after leaving the plasma has not been taken into account in the model. Previous measurements of the transport parameters have typically relied on spectroscopic measurements of $\Delta n=0$ transitions or on $\Delta n=1$ transitions in the K-shell. ${ }^{27-30,36-30}$ Our investigation thus demonstrates that L-shell spectroscopy can be added to the list of methods which can be employed to make measurements of the transport parameters.

The modelling calculations can be checked for consistency by comparing the calculated radial charge-state distribution to the measured profiles. Figure 22(a) shows the sightline-integrated intensity profile of the resonance line $3 C$ of neonlike selenium measured with the new USX spectrometer. The data were obtained by poloidally scanning the spectrometer on a shot-toshot basis and consist of 18 points from 18 reproducible discharges. Each data point reptesents a time average of 16 msec recorded at $t=44-60$ msec after injection. An adjustment is made for the amount of selenium injected into the plasma by normalizing the data to the peak of the energy-integrated soft $x$-ray signal from a soft $x$-ray diode array. Due to the low ratio of plasma pressure to magnetic pressure of the PLT plasmas we assume concentric magnetic flux surfaces and thus poloidal symmetry. Consequently, we symmetrize the profile data of Fig. 22(a) around the origin by setting $f\left(-R_{\text {TAN }}\right)=f\left(R_{\text {TAN }}\right)$, where $R_{\text {TAN }}$ is the distance between the sightline 
and the center of the plasma. The result is shown in Fig. 22(b). Here we have also fitted the symmetrized data using cubic splines. The profile obtained in the spline fit is then Abel-inverted, ${ }^{38,40}$ and the resulting local emissivity profile is shown in Fig. 22(c). The local emissivity profile is compared to the emissivity profile calculated by the MIST code for $t=50$ msec after injection. In the calculations the parameter values determined above, i.e., $D=5 \times 10^{3} \mathrm{~cm}^{2} / \mathrm{sec}$ and $c_{v}=0$, were used. The calculated emissivity profile of the neonlike resonance line $3 C$ is shown in Fig. 23(a) together with the measured emissivity profile. We note that the MIST predictions as well as measurements show that an approximate equilibrium in the relative chargestate abundances is reached within the overall impurity density decay for $t Z$ 40 msec after injection. Hence the profile shapes shown in Fig. 22(c) do not appreciably change later in time. Emissivity profiles have also been measured for the sodiumlike resonance transition $\left(2 p_{1 / 2}^{5} 3 s_{1 / 2} 3 d_{3 / 2}\right)_{3 / 2} \rightarrow\left(2 p^{8} 3 s_{1 / 2}\right)_{1 / 2}$, labelled $N 47$ in Fig. 14, and the sum of the fluorinelike resonance transitions $\left(2 p_{1 / 2} 2 p_{3 / 2}^{3} 3 d_{3 / 2}\right)_{3 / 2} \rightarrow 2 p_{3 / 2}^{5}$ and $\left(2 p_{1 / 2} 2 p_{3 / 2}^{3} 3 d_{3 / 2}\right)_{6 / 2} \rightarrow 2 p_{3 / 2}^{5}$. These transitions are labeiled $F 55$ and $F 56$, respectively, in Fig. 14. The profiles are shown in Figs. 23 (a) and (b), respectively, along with the profiles calculated by the MIST code. The scales of all experimental and calculated profiles have been normalized to the peak value of the measured intensity of line $3 C$. In order to get agreement between the relative magnitudes of the computed and experimental profiles seen in Figs. 23 (a)-(c), it was necessary to increase the ionization rate of the sodiumlike ions by a factor of 1.8 in the calculations. Without increasing the ionization rate of sodiumlike ions the scale of the computed profile of the sodiumlike emissivity would have been 1.8 times larger than the experimental profile, although its shape would have been the same. Since the ionization and recombination rates of Post et al ${ }^{32}$ employed in the MIST code are deemed uncertain by factors of two to four, ${ }^{32}$ an adjustment of one of the rates by a factor of 1.8 is within reason. This is particularly true because these ionization rates do not account for inner shell ionization, which is an important mechanism for sodiumlike ions. ${ }^{41,42}$ By contrast, the excitation rates and branching ratios which we have used in the calculation of the line emissivities are deemed much more accurate. 
The location of the peak as well as the gross shape of the line emission profile are mostly determined by the electron temperature profile. On the other hand, the wings of the line errission (i.e., the radial gradient of the profile) are sensitive to the value of the transport parameters. For a reliable determination of the transport parameters it is, therefore, necessary to measure the line intensity of a given line not only in the central plasma region where the intensity is strongest, but also in the colder regions of the plasma where the intensity is weak. Such a measurement was easily possible with our new spectrometer, as seen in Fig. 22, due to the good sensitivity of the detector and because the brightness of neonlike transitions. Comparing the experimental data with the shapes of the modelling profiles in Fig. 23, we find good agreement. Hence, we have been able to construct a consistent model which, within the uncertainties of the ionization and recombination rates, matches both the temporal and spatial line emission profiles measured with the USX spectrometer and which provides us with a measure of the magnitude of plasma transport. 


\section{Acknowledgments}

We are grateful to Ray Fonck, Mark Eckart, and John Boychuck for providing us with useful advice in the design of the new spectrometer. Mike Dixon, John Gray, and co-workers have built the detector assembly at Galileo Electro-Optics Corporation in Sturbridge, Massachussetts. We wish to thank Jin Gorman, Jim Lehner, Steve Styner, and Phil Howard for their assistance in assembling the spectrometer, as well as Greg Lemunyan who built the filter and suppressor boards. Janet Felt developed most of the software for the data acquisition. We also thank the members of the PLT operating crew for their cooperation and for providing the observed plasma discharges.

We are grateful for the support of Lowell Wood, Art Toor, Joel Hosea, Harold Furth, and Ken Young. Funding was received from the U. S. Department of Energy under contracts No.DE-ACO2-76-CHO-3073 and W-7405ENG-48 and from Lawrence Livermore National Laboratory under subcontracts SANL-622-033 and P.O. 8-668-705. One of the authors (P.B.) was supported by the Fannie and John Herts Foundation during most of this work. 


\section{References}

${ }^{1}$ E. Hinnov, in Atomic and Molecular Processes in Controlled Thermonuclear Fusion, ed. by M. R. C. McDowell and A. M. Ferendeci (Plenum Press. New York, 1980), p. 449.

${ }^{2}$ C. de Michelis and M. Mattioli, Nucl. Fusion 21, 677 (1981).

3M. Bitter, S. von Goeler, M. Goldman, K. W. Hill, R. Horton, W. Roney, N. Sauthoff, and W. Stodiek, in Temperature, its Measurement and Control in Science and Industry, edited by S. F. Schooley (American Institute of Physics, New York, 1982), Vol. 5, p. 693.

${ }^{4}$ K. W. Hill, S. von Goeler, M. Bitter, L. Campbell, R. D. Cowan, B. Fraenkel, A. Greenberger, R. Horton, J. Hovey, W. Roney, N. R. Sauthoff, and W. Stodiek, Phys. Rev. A 19, 1770 (1979).

${ }^{5}$ P. Plata, J. Ramette, E. Belin, C. Bonnelle, and A. Gabriel, J. Phys. E 14, 448 (1981).

${ }^{6}$ R. Bartiromo, R. Giannella, M. L. Apicella, F. Bombarda, S. Mantovani, and G. Pizzicarolli, Nucl. Instrum. Methods 225, 378 (1984).

${ }^{7}$ R. Burhenn and H.-J. Kunze, J. Phys. E. 20, 1258 (1987).

'E. Källne and J. Källne, Rev. Sci. Instrum. 58, 1077 (1987).

${ }^{9}$ R. J. Fonck, A. T. Ramsey, and R. V. Yelle, Appl. Opt. 21, 2115 (1982).

${ }^{10}$ J. L. Schwob, A. W. Wouters, S. Suckewer, and M. Finkenthal, Rev. Sci. Instrum. 58, 1601 (1987).

${ }^{11}$ H. H. Johann, ¿̇. Phys. 69, 185 (1931).

${ }^{12}$ P. Beiersdorfer, M. Bitter, S. von Goeler, R. Bell, A. Cavallo, S. Cohen, K. Hill, R. Hulee, J. Timberlake, R. Walling, B. Whitten, and P. Hagelstein, Bull. Am. Phys. Soc. 31, 1468 (1986). 
${ }^{13}$ A. Burek, Space Ści. Instrum. 2, 53 (1976).

${ }^{14}$ G. Brogren, Arkiv Fysik 3, 515 (I951).

${ }^{15} \mathrm{R}$. J. Fonck in ACS Symposium Series, No. 236, Multichannel Image Detectors, edited by Y. Talmi, (American Chemical Society, New York, 1983), Vol. 2, p. 277.

${ }^{16}$ Galileo Electro-Optics Corp., Galileo Park, Sturbridge, MA 01518.

${ }^{37}$ B. L. Henke, J. P. Knauer, and K. Premaratne, J. Appl. Phys. 52, 1509 (1981).

${ }^{16}$ EG\&G Reticon, 345 Potrero Ave., Sunnyvale CA 94086.

${ }^{10}$ H. W. Schnopper and K. Kalata, Appl. Phys. Lett. 15, 134 (1969).

${ }^{20}$ Perkin Elmer, Physical Electronics Division, 6509 Flying Cloud Dr., Eden Prairie, MN 50344.

${ }^{21}$ J. L. Wiza, Nucl. Instrum. Methods 182, 587 (1979).

${ }^{22}$ EG\&G Datasheet S-Series Solid State Line Scanners - 128, 512, and 1024 Elements, EG\&G Reticon, 345 Potrero Ave., Sunzyvale CA 94086.

${ }^{23}$ Hamamatsu Technical Manual No. RES-0795, Hamamatsu Corp., 420 South Ave., Middlesex, NJ 08836.

${ }^{24}$ R. J. Fonck, private communication (1985).

${ }^{23}$ E. S. Marmar, J. L. Cecchi, and S. A. Cohen, Rev. Sci. Instrum. 46, 1149 (1975).

${ }^{20}$ J. Timberlake, S. Cohen, C. Daughney, and D. Manos, J. Vac. Sci. Technol. A 1, 841 (1983).

${ }^{27}$ S. A. Cohen, J. L. Cecchi, and E. S. Marmer, Phys. Hev. Lett. 35, 1507 (1975). 
${ }^{25}$ E. S. Marmar, J. E. Rice, and S. L. Allen, Phys. Rev. Lett. 45, 2025 (1980).

29E. S. Marmar, J. E. Rice, J. L. Terry, and F. H. Seguin, Nucl. Fusion 22, 1567 (1982).

${ }^{30}$ TFR Group, Nucl. Fusion 23, 559 (1983).

${ }^{31}$ R. A. Hulse, Nucl. Technol. Fusion 3, 259 (1983).

${ }^{32}$ D. E. Post, R. V. Jensen, C. B. Tartar, W. H. Grasberger, and W. A. Lokke, At. Data Nucl. Data Tables 20, 397 (1977).

${ }^{30} \mathrm{R}$. A. Hulse, in Proc. of TFTR/JET INTOR Workshop on Plasma Transport, ed. by C. E. Singer; Princeton Plasma Physics Laboratory Report No. PPPL-2128 (1984).

34. Bar-Shalom, M. Klapisch, and J. Oreg, Phys. Rev. A (1988) [in press],

${ }^{35}$ M. H. Chen, Phys. Rev. A 34, 1073 (1986).

${ }^{30}$ R. J. Fonck, M. Finkenthal, R. J. Goldston, D. L. Herndon, R. A. Hulse, R. Kaita, and D. D. Meyerhofer, Phys. Rev. Lett. 48, 737 (1982).

${ }^{37}$ R. C. Isler, L. E. Murray, E. C. Crume, C. E. Bush, J. L. Dunlap, P. H. Edmozds, S. Kasai, E. A. Lazarus, M. Muralami, G. H. Neilson, V. K. Paré, S. D. Scott, C. E. Thomas, and A. J. Wootton, Nucl. Fusion 23, 1017 (1983).

${ }^{30}$ R. J. Fonck and R. A. Hulse, Phys. Rev. Lett. 52, 530 (1984).

${ }^{30}$ K. Boclasten, J. Opt. Soc. Am. 51, 943 (1961).

${ }^{40}$ W. L. Barr, J. Opt. Soc. Am. 52, 885 (1962).

${ }^{41}$ D. H. Sampeon and H. Zhang, Phys. Rev. A 36, 3590 (1987).

12P. Beiersdorfer, Ph.D. thesis, Princeton University (1988).

4F. Biggs and R. Lighthill, Sandia Laboratories Report No. SC-RR-71 0507, 1971. 


\section{Figures}

FIG. 1. Focussing properties of the Johann geometry. The Johann geometr? employs a bent crystal with a radius of curvature $R_{c}$ to focus $\mathrm{x}$ rays of wavelength $\lambda$ on to a circle with radius $R_{c} / 2$, the so-called Rowland circle. According to Bragg's law $x$ rays of different wavelengths are focussed on different points along the Rowland circle.

FIG. 2. Schematic of the new vacuum crystal spectrometer.

FIG. 3. Absorption characteristics of the aluminum and the magnesiumparylene foils according to Biggs and Lighthil in Ref. 43. $\mu$ is the linear absorption coefficient, and $d$ is the thickness of the foil. Absorption edges occur at $284 \mathrm{eV}$ for ccsbon, $50 \mathrm{eV}$ and $1305 \mathrm{eV}$ for magnesium, and 73 $\mathrm{eV}$ and $1560 \mathrm{eV}$ for aluminum.

FIG. 4. Schamatic of the intensified soft $x$-ray detector.

FIG. 5. Block diagram of the electronics used to read out and archive the intensified photodiode array.

FIG. 6. Side view of the four-bar bending jig.

FIG. 7. Crystal focus determined via optical means for different distances $\Delta x$ from best focus: (a) $\Delta x=+0.9 \mathrm{~cm}$, (b) $\Delta x=+0.5 \mathrm{~cm}$, (c) $\Delta x=+0.3$ $\mathrm{cm}$, (d) $\Delta x=-0.3 \mathrm{~cm},(\mathrm{c})$,(f) $\Delta x=-1.2 \mathrm{~cm}$. The illuminated length of the crystal in (a)-(e) is $2 \mathrm{~cm}$; in (f) it is $5 \mathrm{~cm}$. Note the change of scale betwoen (a)-(d) and (e), (f).

FIG. 8. Quality of the crystal focus as a function of distance for two ADP crystals: (a) 0.500 mm-thick crystal, polished on both sides; (b) $0.25 \mathrm{~mm}$ thick crystal, polished on one side only. The illuminated length of each crystal is $2 \mathrm{~cm}$. The solid line is a theoretical estimnte of the focal width using Eq. (6) with $R_{c}=57.3 \mathrm{~cm}$ and $\theta=50^{\circ}$.

FIG. 9. Detector response as a function of (a) the microchannel plate voltage,

(b) the focussing potential on the phosphor. 
FIG. 10. Spectrum of the Lyman series in hydrogenic sodium. The spectrum covers the wavelength region 7.50-8.15 $\AA$, and has been obtained with the vacuum crystal spectrometer ou PLT. The spectrum is due to transitions of the type $n P_{1 / 2,3 / 2} \rightarrow 1 S_{1 / 2}$ for $n=4,5,6,7 \ldots$ The intensity of the Ly- $\zeta$ line at $\lambda=7.6767 \AA$ is enhanced by charge-exchange recombination between neutral hydrogen and fully stripped sodium ions. This process populates preferrentially the $n=7$ level. ${ }^{36}$

FIG. 11. Line width as a function of microchannel plate voltage. The different symbols correspond to different spectral lines. The potential on the phosphor is $2400 \mathrm{~V}$.

FIG. 12. Line widths of two different lines as a function of the accelerating potential between the back of the Chevron and the phosphor screen. The voltage across the microchannel plates is $1300 \mathrm{~V}$.

FIG. 13. Line width as a function of line intensity. The detector voltages are $V_{M C P}=1300 \mathrm{~V}$ and $V_{P H}=2450 \mathrm{~V}$.

FIG. 14. Spectrum of the $3 d \rightarrow 2 p$ transitions in fluorine-, neon- and sodiumlike selenium in the wavelength region $7.30-7.95 \AA$. Note that the widths of the two most intense lines, $3 C$ and $3 D$, are larger than those of the weater lines.

FIG. 15. Raw data scan showing systeraatic noise patterns. Every fourth pixel has a common offset. Cf. Fig. 14 which shows the same data after numerical elimination of the offset pattern.

FIG. 16. Intensity response of the detector obtained with Reticons \# 1 and \# 2. The response function was obtained by sweeping the detector over a constant source of monoenergetic $x$ rays.

FIG. 17. Position of the crystal and the detector for the two nominal Bragg angles, $\theta_{0}$ and $\theta_{0}-\Delta$. The detector can be kept in alignment on the Rowland circle by tilting the detector arm around $P$. The sight line between the crystal and the plasma remains fixed. 
FIG. 18. Tilt mechanism between crystal and detecuur used to keep the detector in focus on the Rowland circle when changing the spectrometer alignment. Nuts $\mathbf{A}$ set the degree of tilting; nuts $\mathbf{B}$ determine the distance between pivot and crystal, as well as between pivot and detecior.

FIG. 19. Ka spectrum of heliumlike argon. The spectrum is measured in second order with the vacuum crystal spectrometer. The resonance line $w$, the intercombination lines $x$ and $y$, and the forbidden line $z$ are labelled. The spectrum is superposed on to a variety of strong transitions seen in first order. These transitions presumably arise from $4 \rightarrow 2$ transitions in berylliumlike and boronlike iron.

FIG. 20. Intensity of line $3 C$ versus time after injection into the plasma. The data are integrated along a line of sight through the center of the plasma.

FIG. 21. Experimental and fitted intensity of line $3 C$ of neonlike selenium for three values of the diffusion coefficient $D$. The value of $c_{v}$ is set $c_{v}=0$. The values of the diffusion coefficient $D$ are given in units of $\mathrm{cm}^{2} / \mathrm{sec}$. The peaks of the calculated intensity curves have been normalized to the peak of the experimental intensity. The selenium is injected at $t=0$.

FIG. 22. (a) Sightline-integrated intensity data of the neonlike transition $3 C$ versus tangency radius. (b) Symmetrized sightline-integrated intensity data of the transition $3 C$ versus tangency radius. The dato are fitted using cubic splines. The dashed curves represent two additional spline fits. (c) Emissivity profile of the neonlike transition $3 C$. The profite is the result of an Abel inversicu of the symmetrized sightline-integrated intensity data in (b). The dashed curves correspond to the dashed profiles shown in (b) and serve to illustrate the uncertainties in the Abel-inverted profiles.

FIG. 23. Calculated and measured emissivity profiles (a) of the neonlike line $3 C$, (b) of the sodiumlike line N47, and (c) of the blend of fluorinelike lines $F 55$ and $F 56$. The transport parameters used in the calculations are: $D=5 \times 10^{3} \mathrm{~cm}^{2} / \mathrm{sec}$ and $c_{v}=0$. 


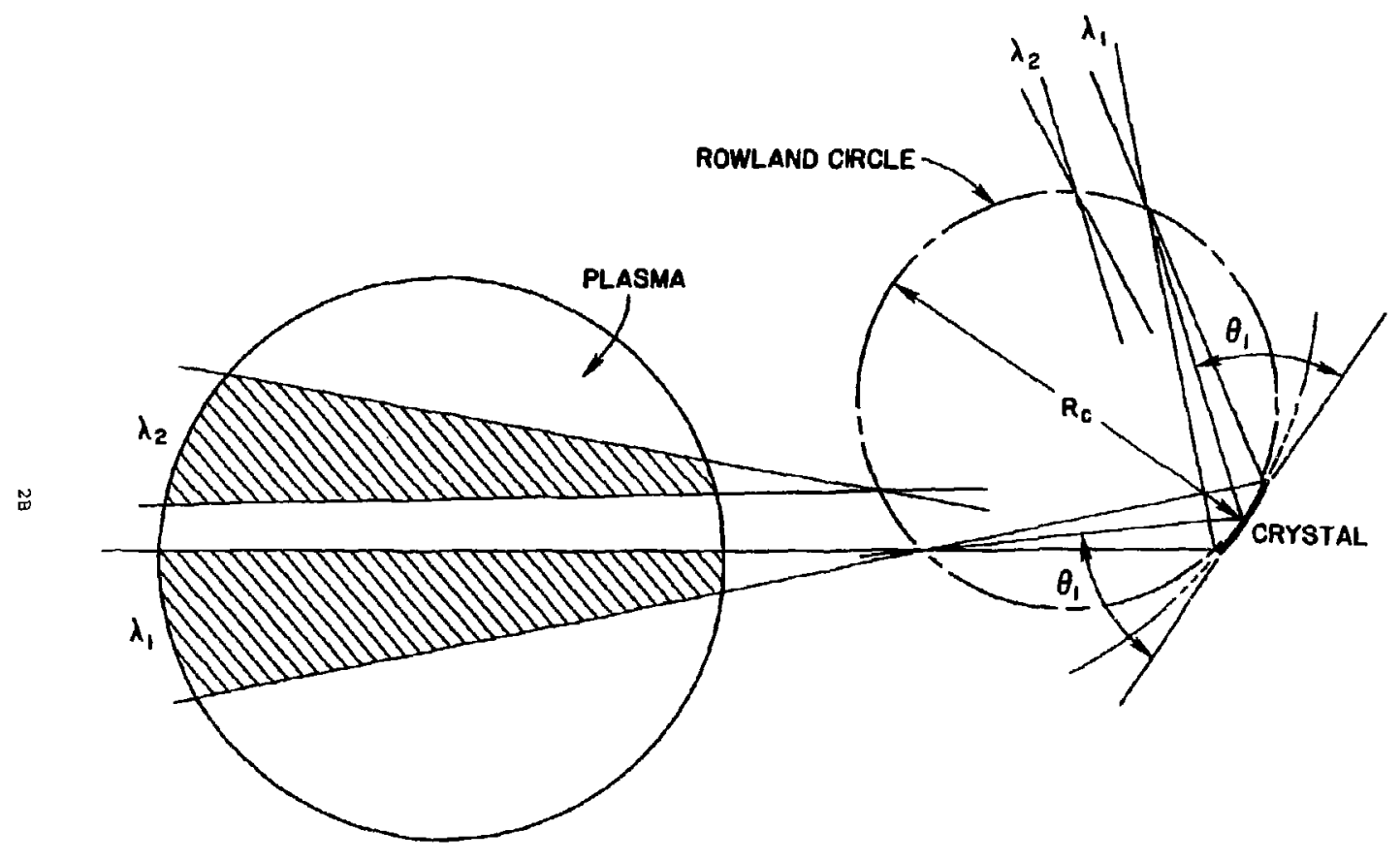

Fig. 1 


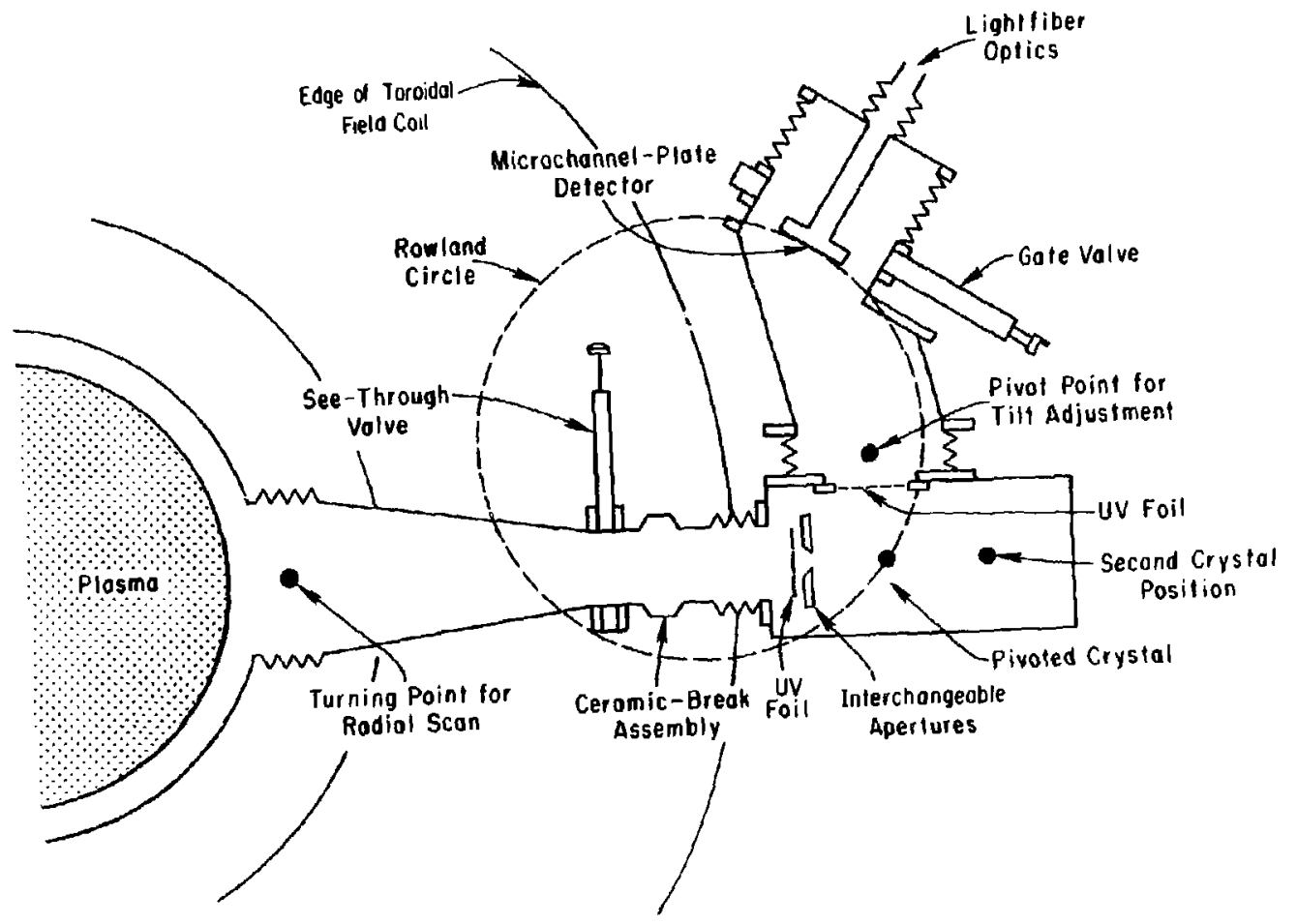

Fig. 2 


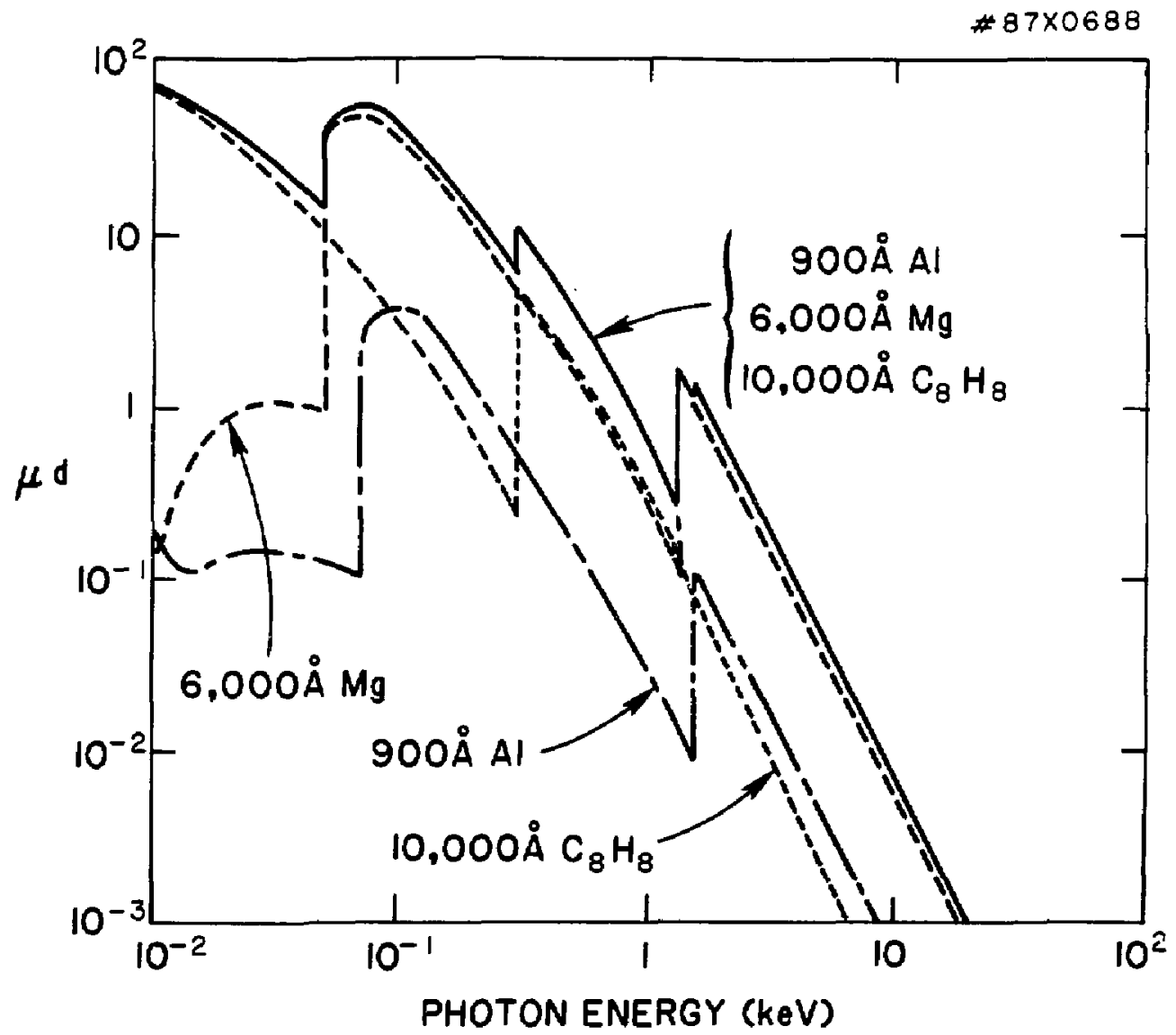

F1g. 3 

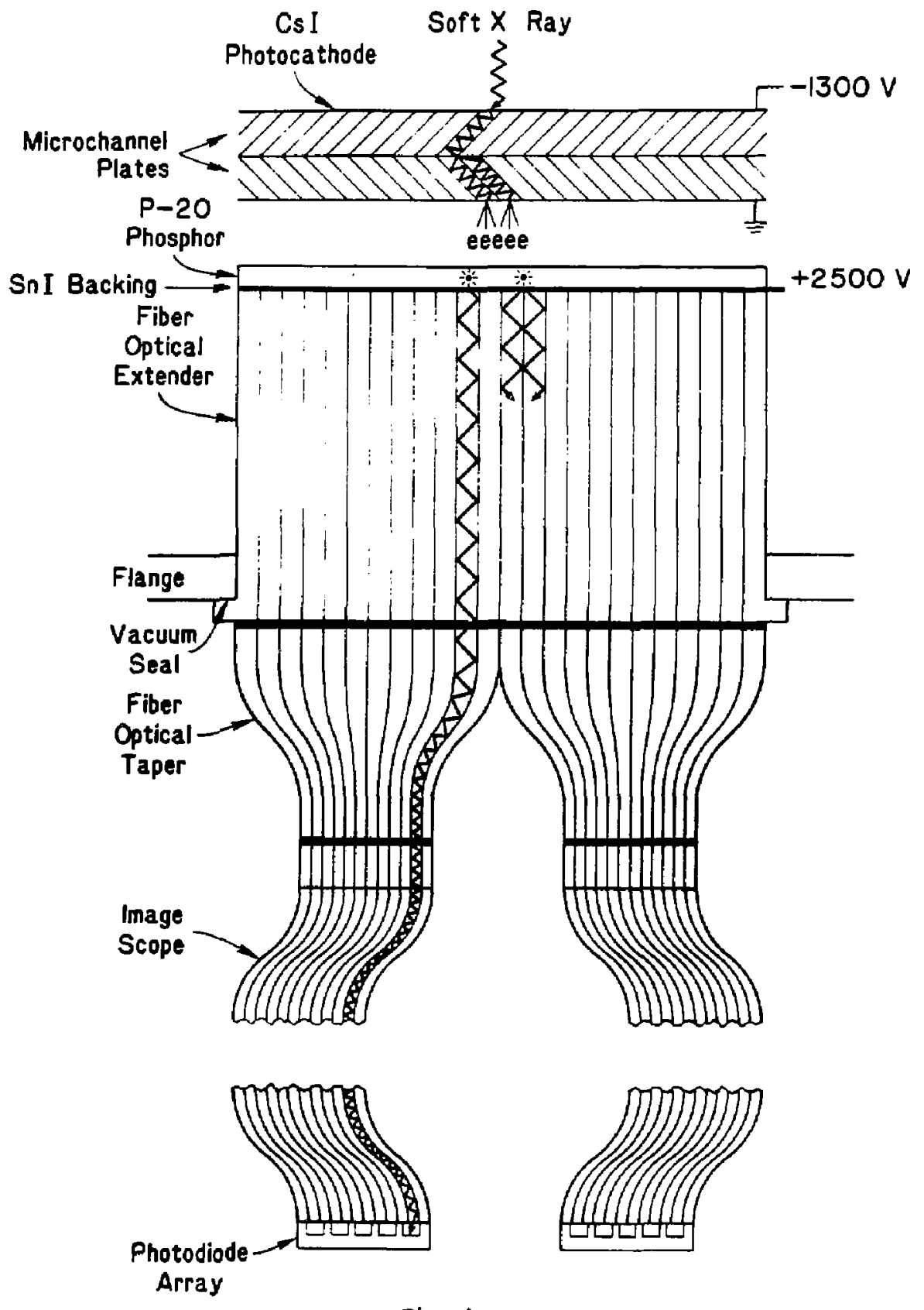

Fig. 4 


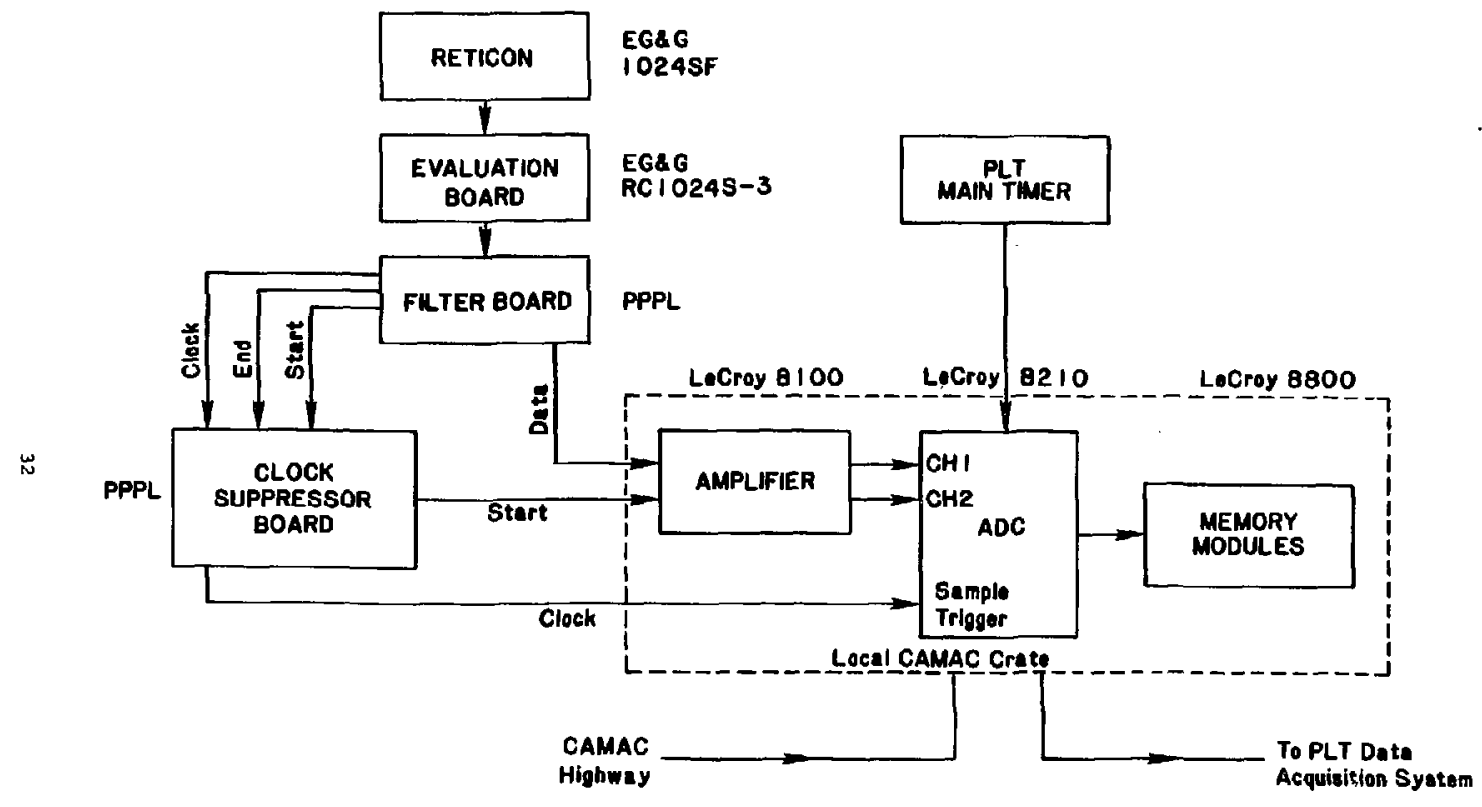

Fig. 5 
\#87X0685

$\omega$

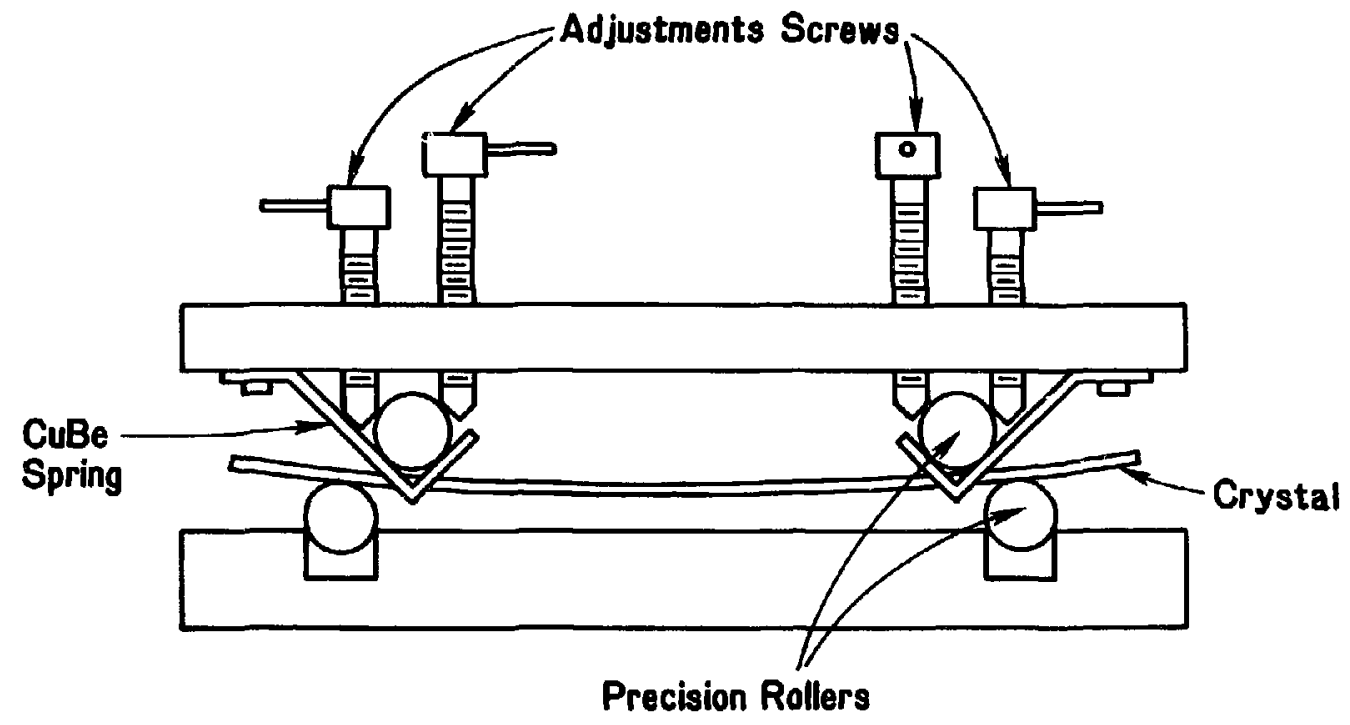

Fig. 6 

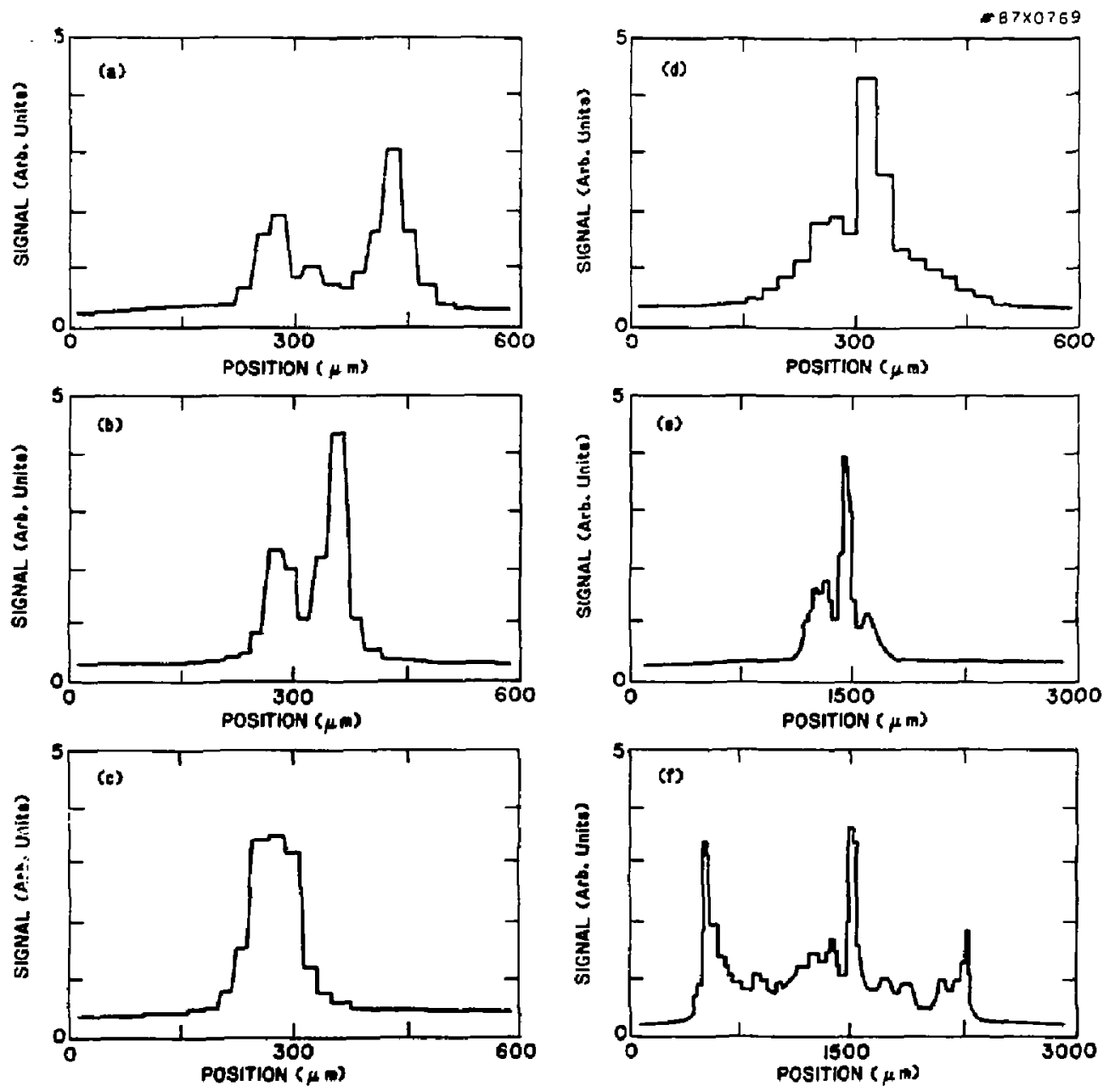

Fig. 7 

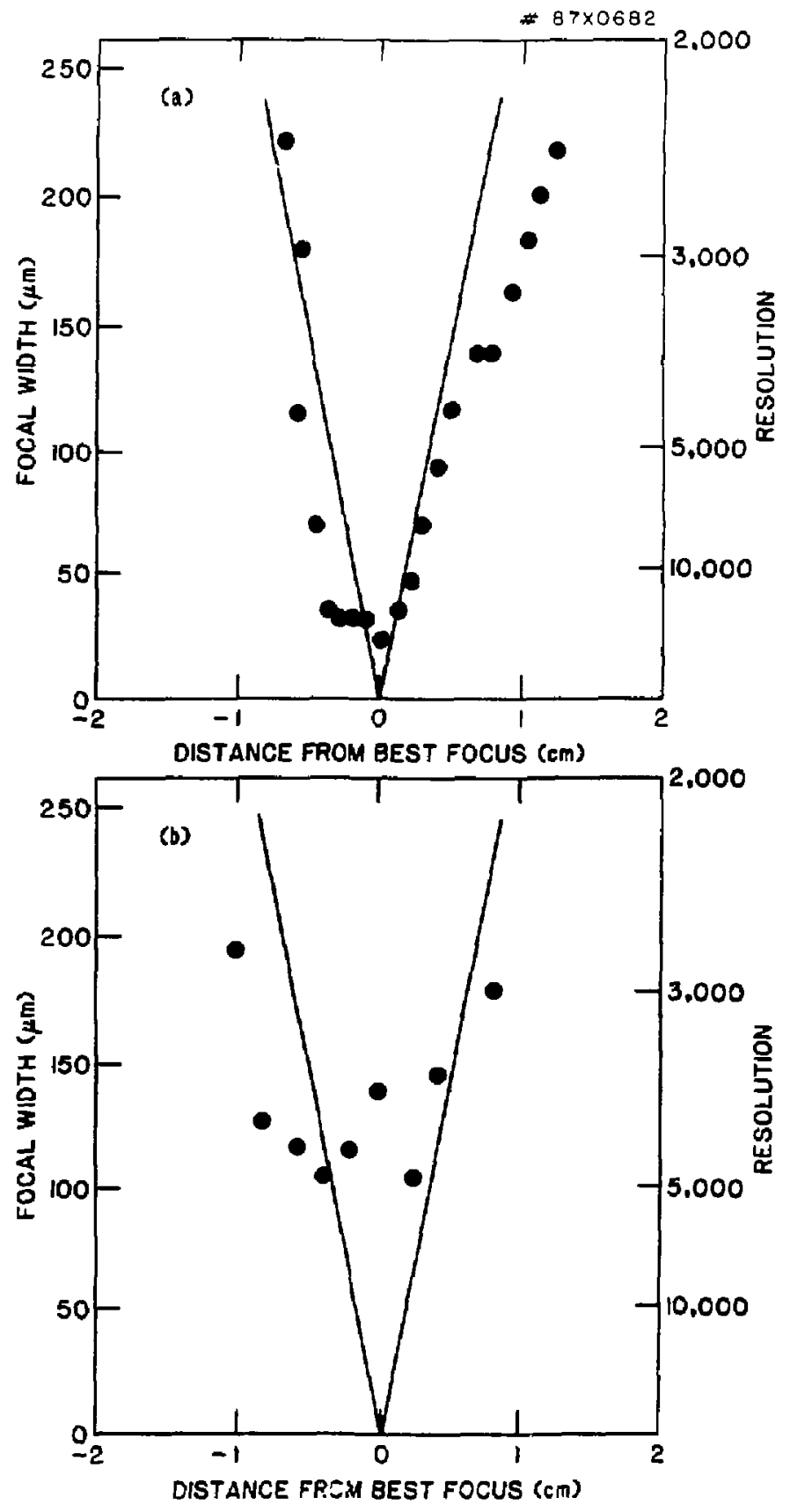

FIg. 8 


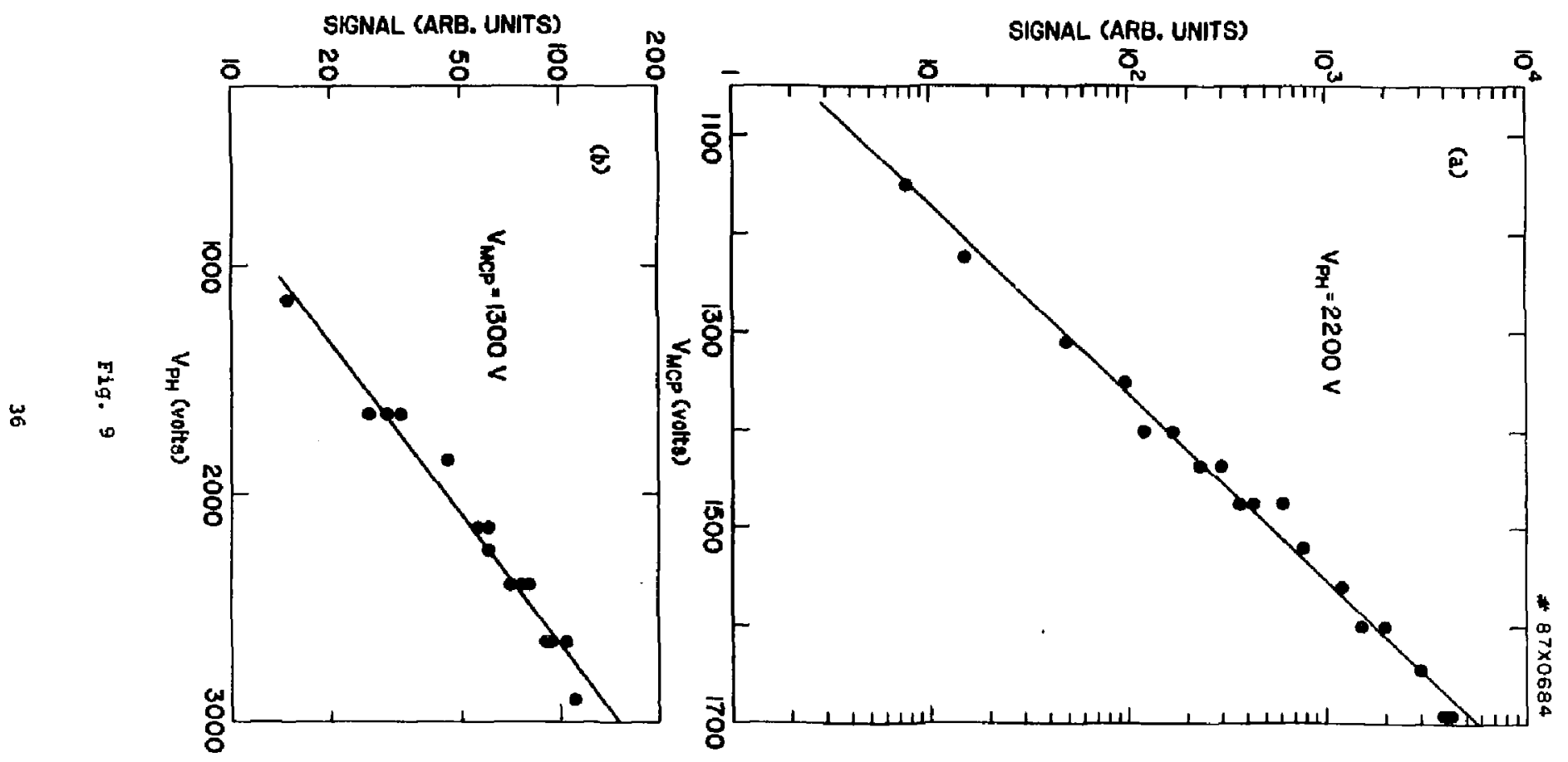




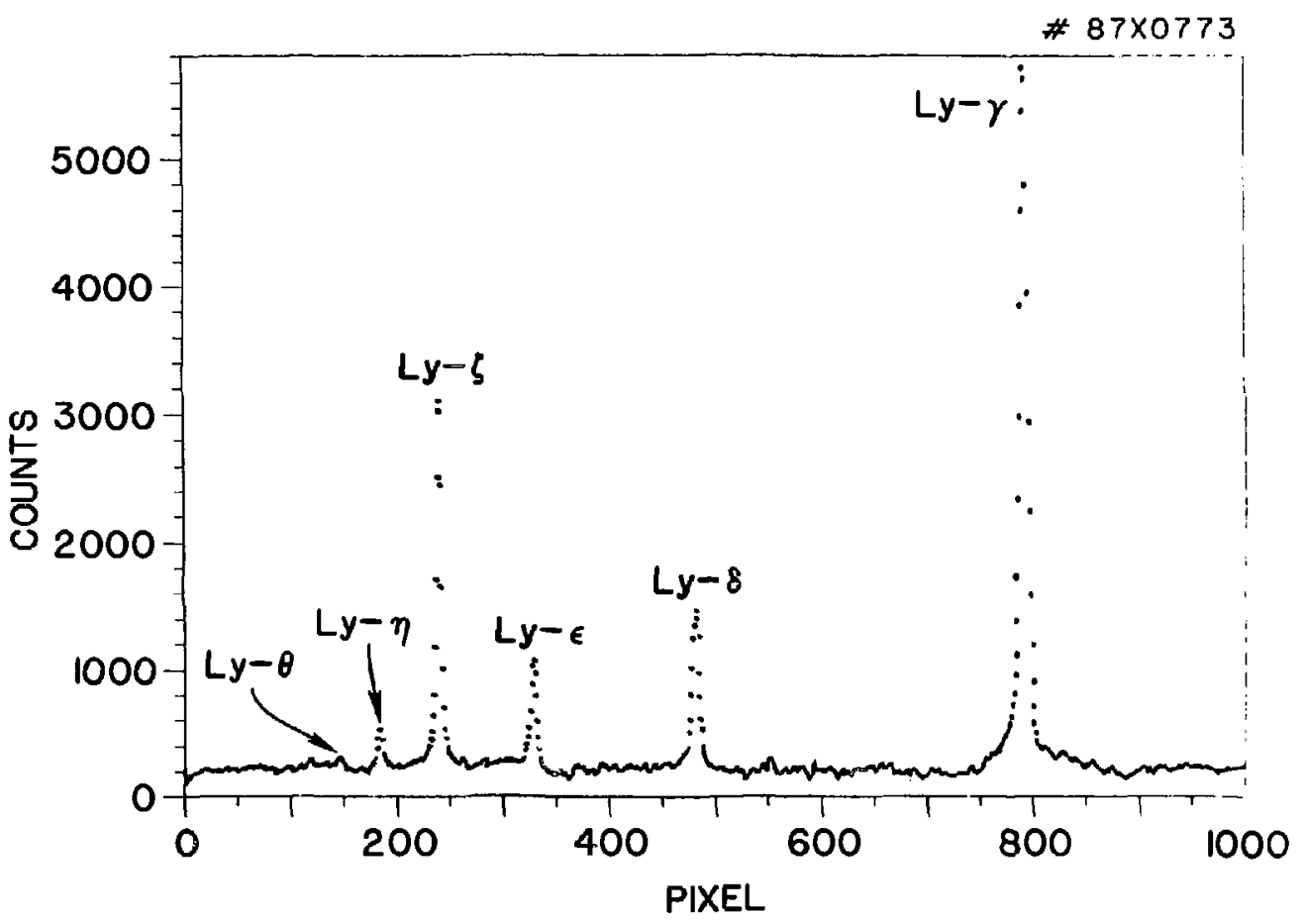

Fig. 10 


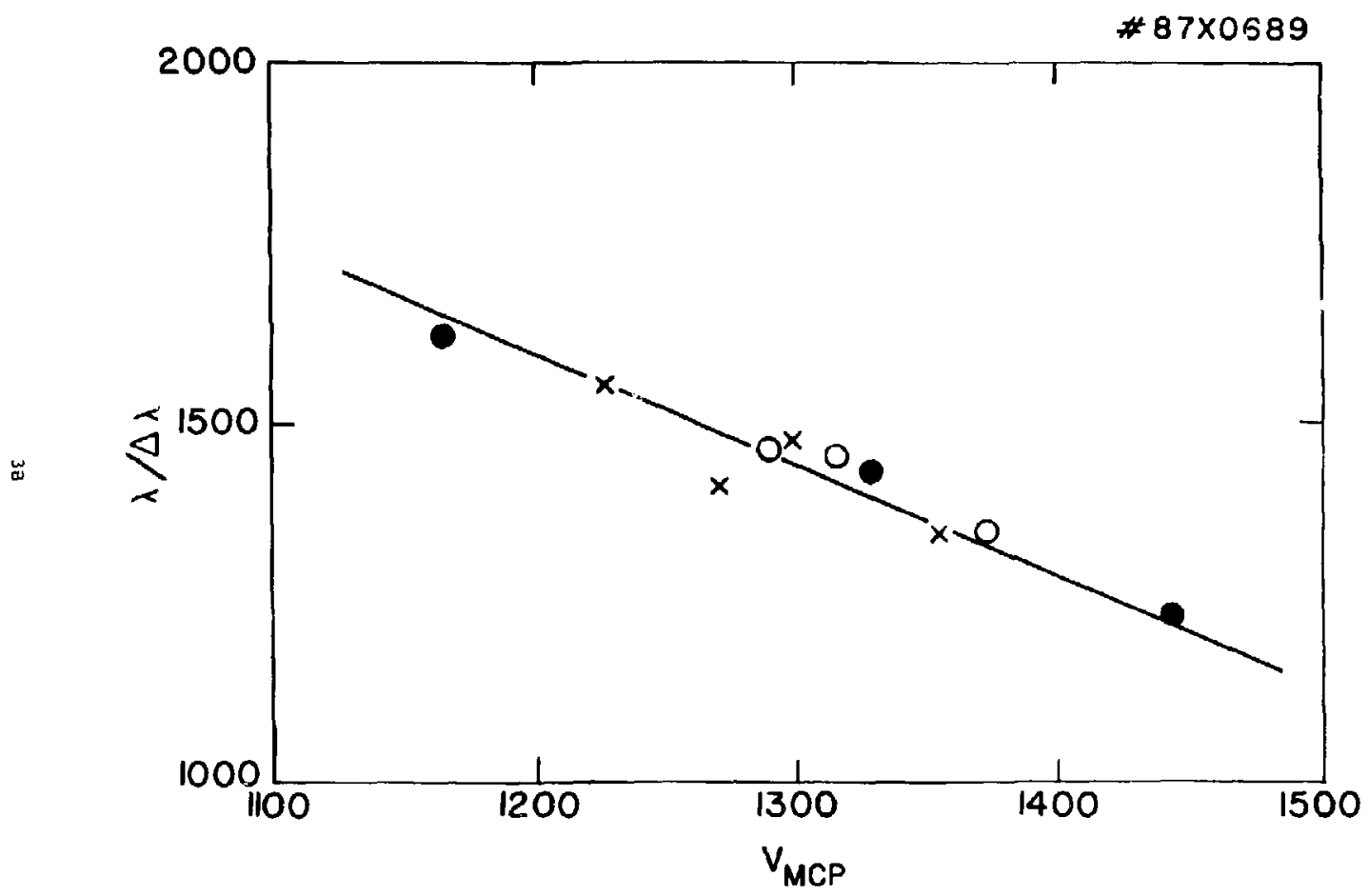

Fig. 11 


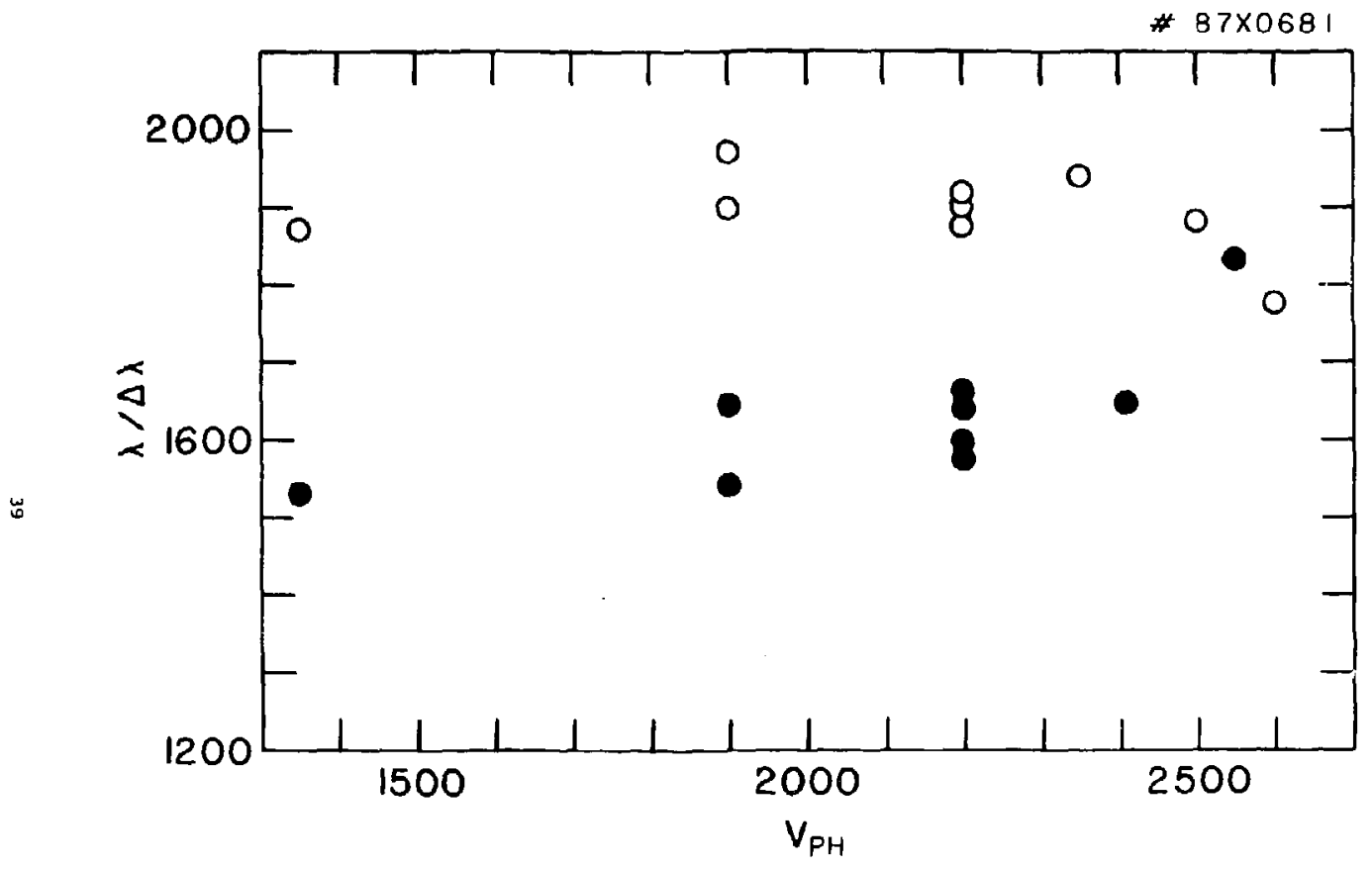

Pig. 12 


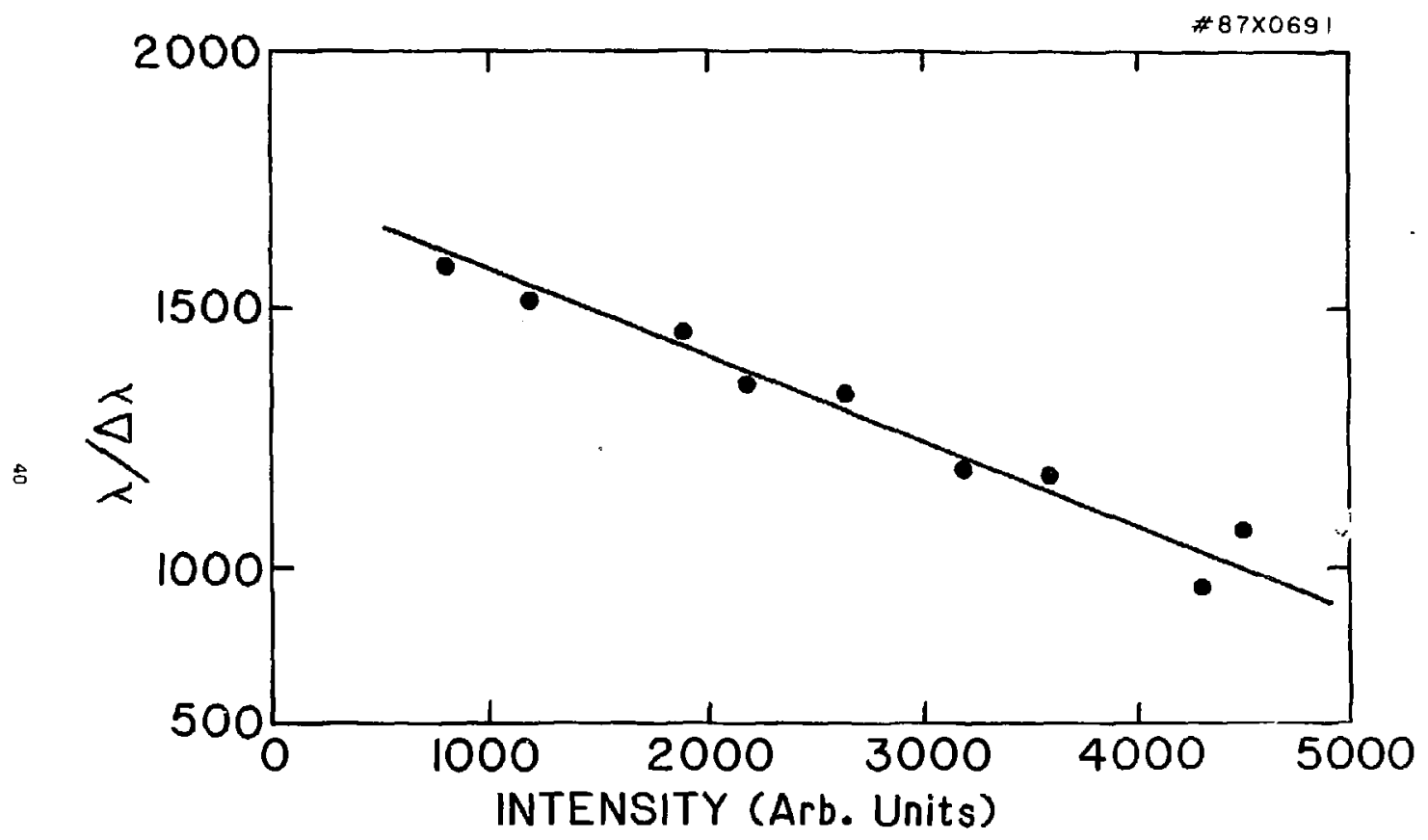

Fig. 13 


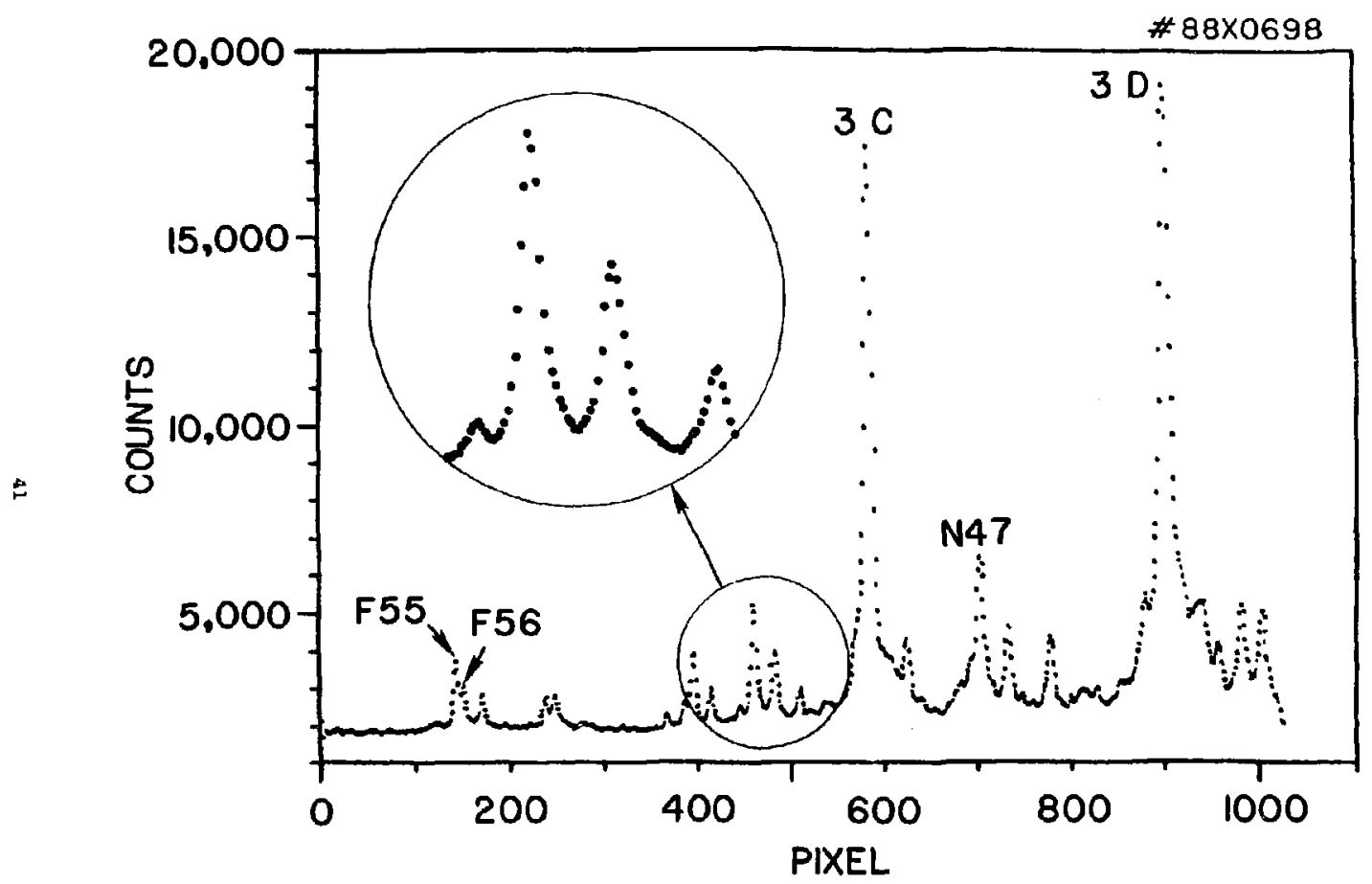

Pig. 14 
\# $87 \times 0774$

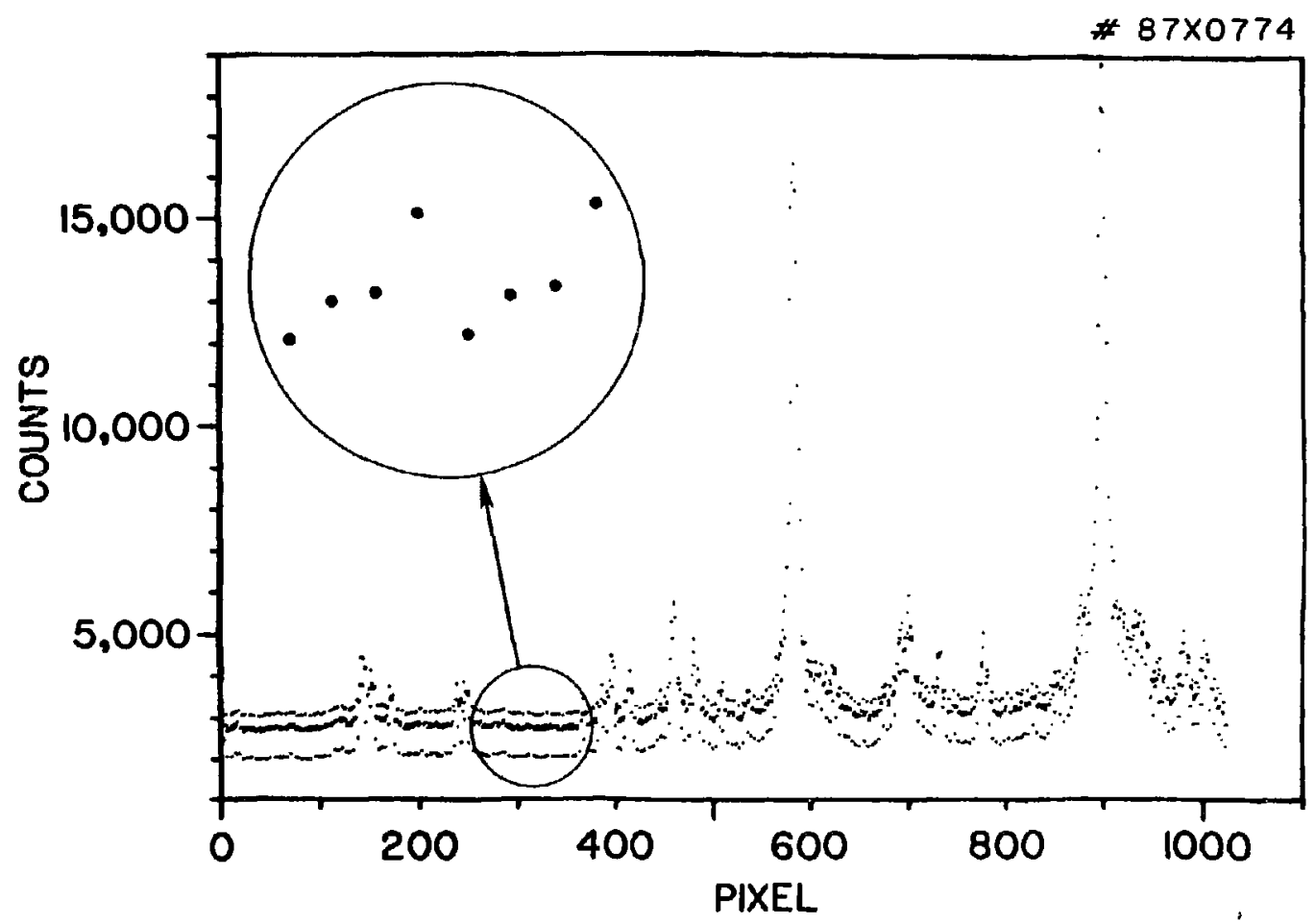

Fig. 15 


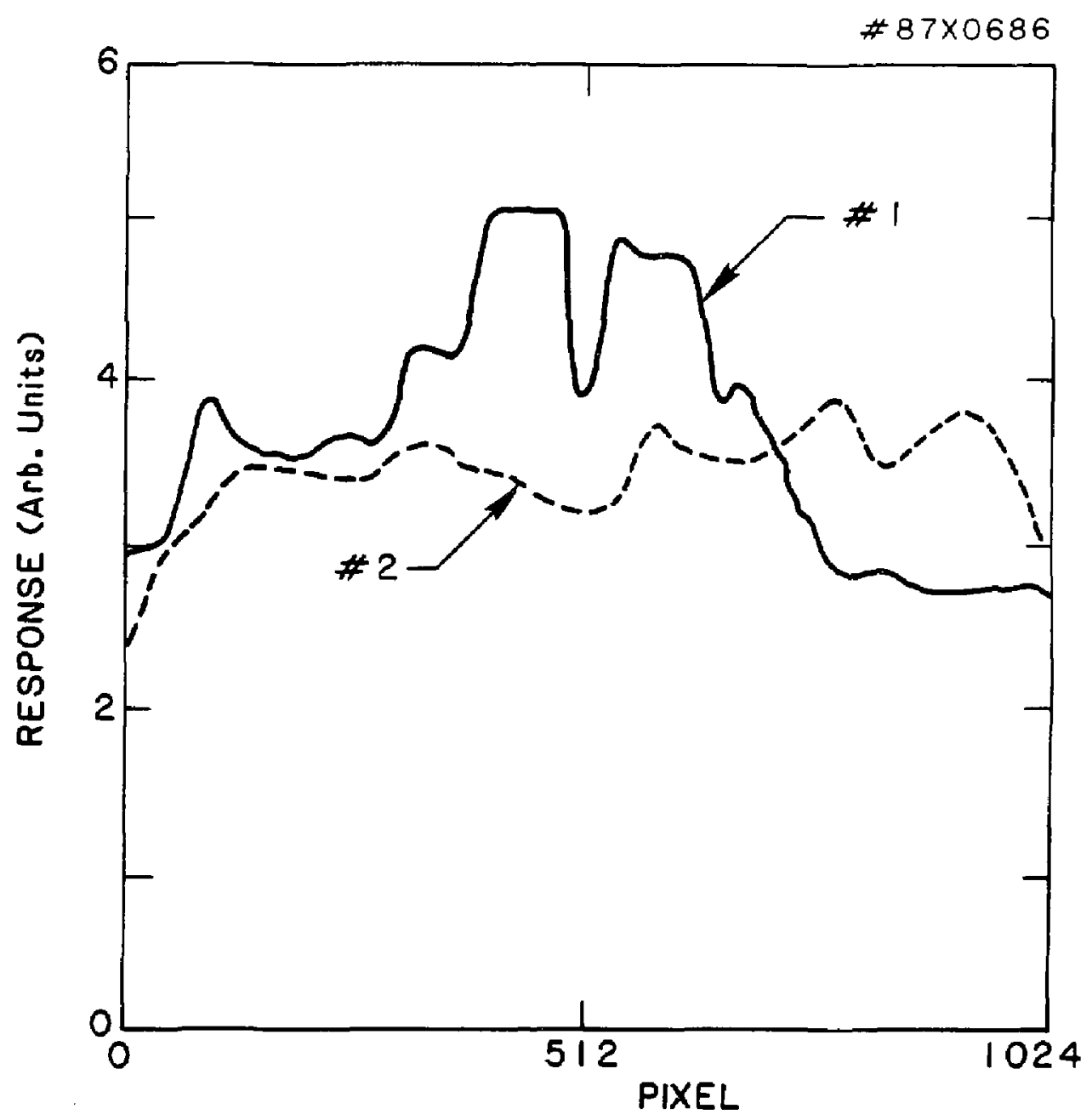

Fig. 16 
\# 87X0741

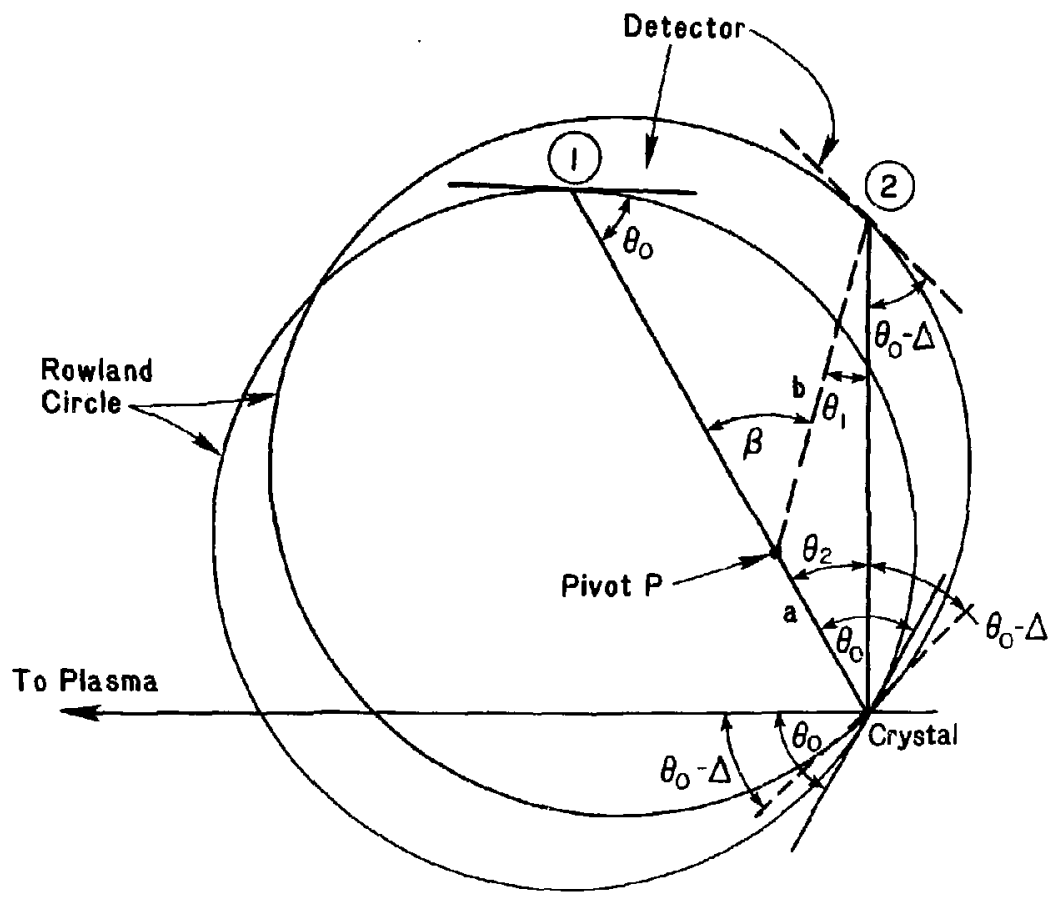

Fig. 17 
\#87X0739

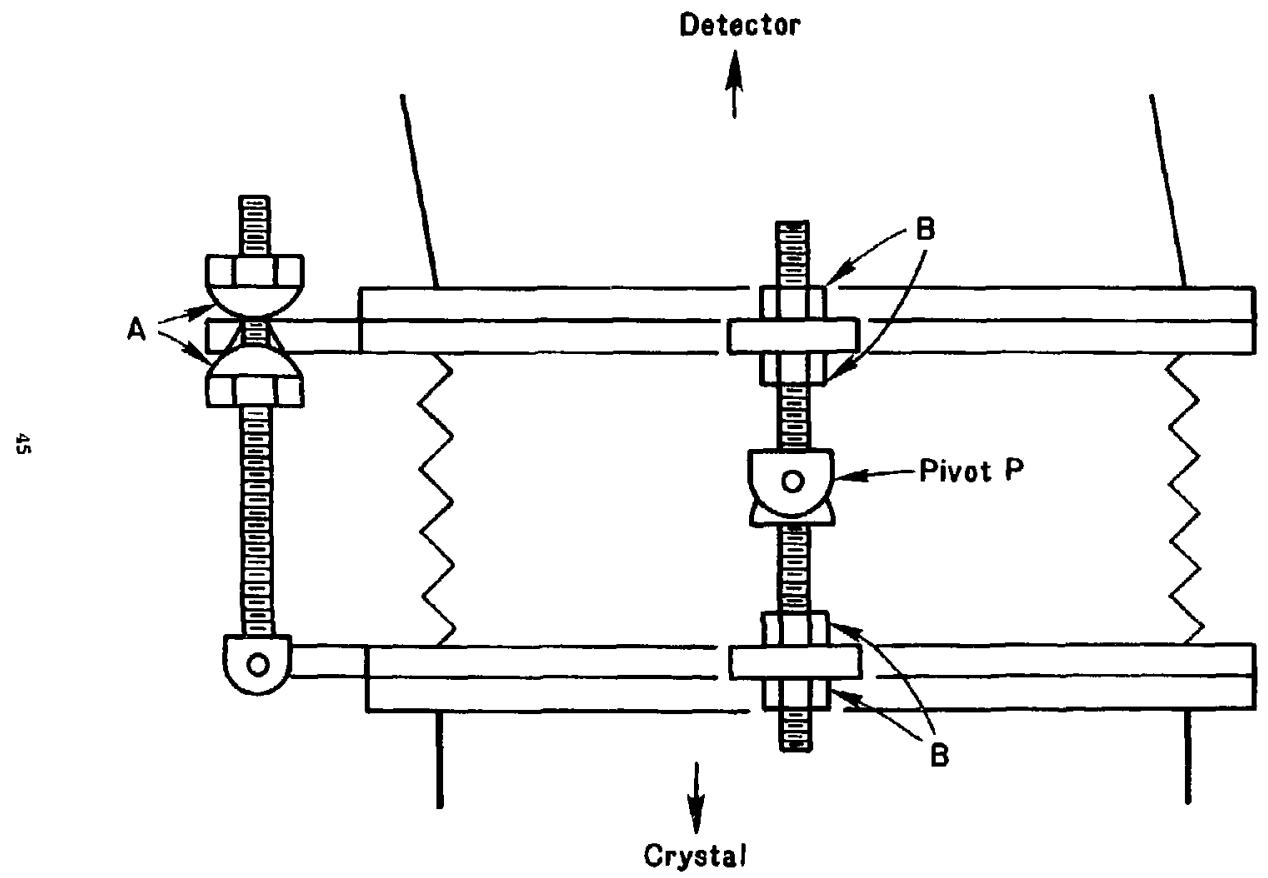

Fig. 18 


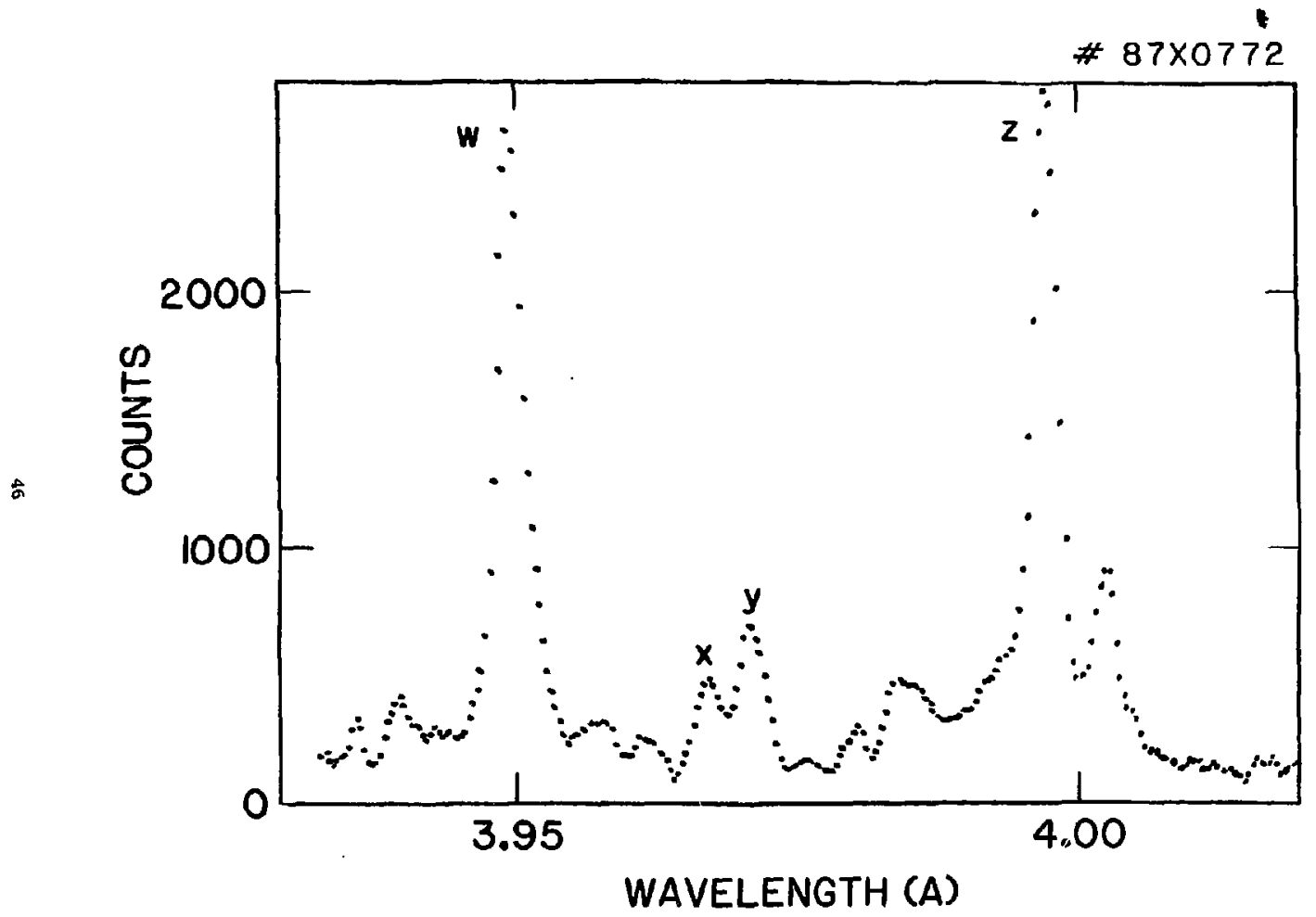

Fig. 19 


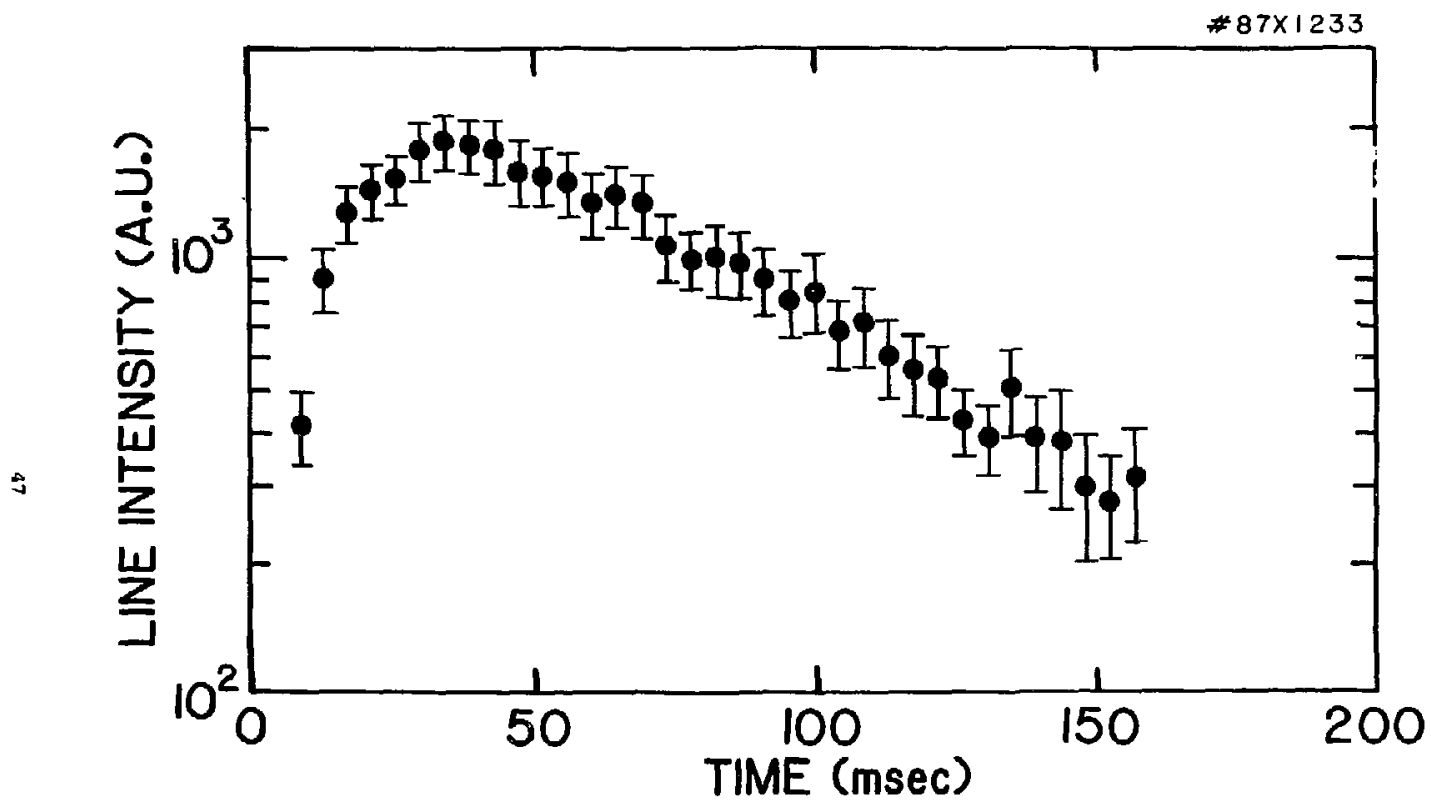

Fig. 20 


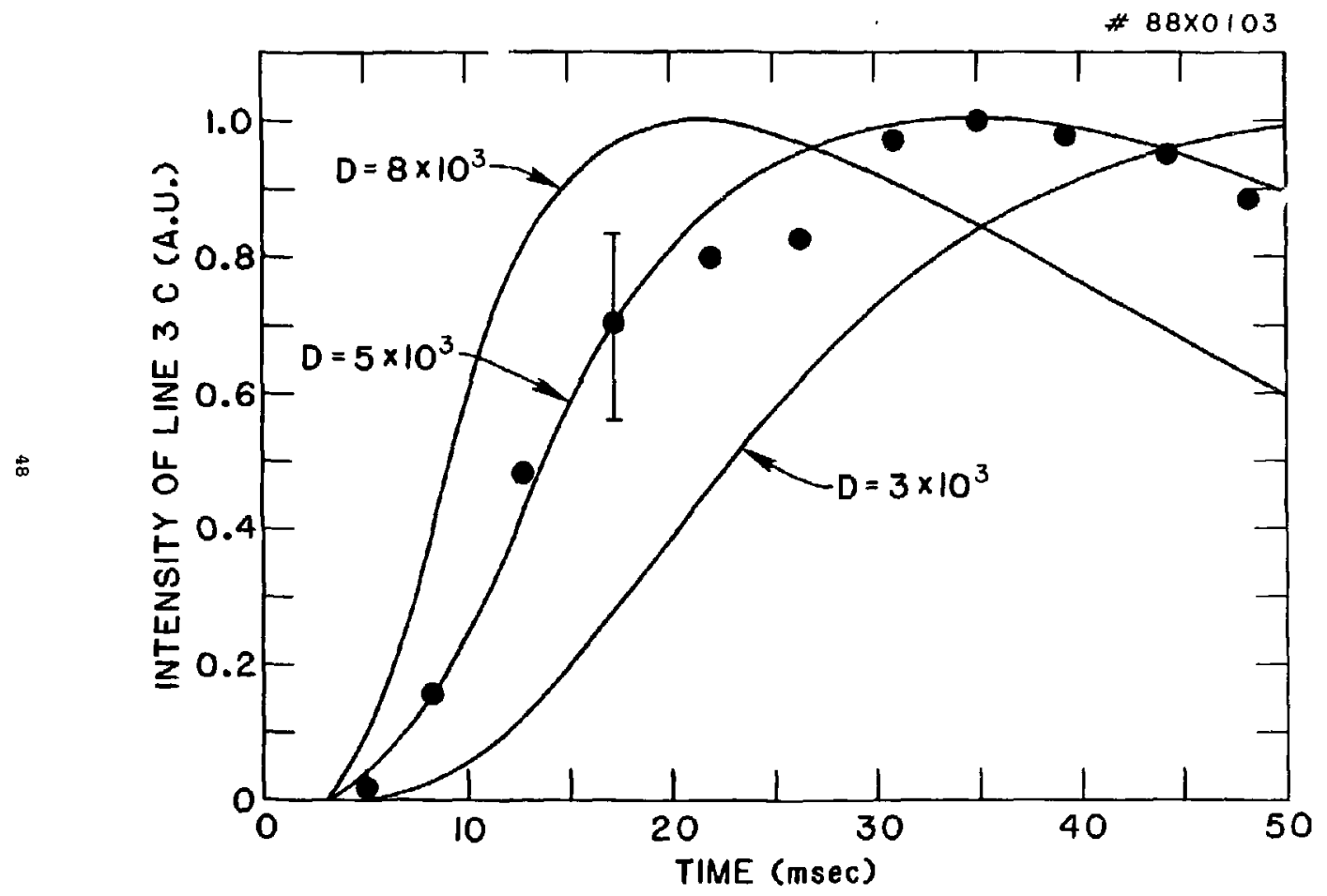

Fig. 21 


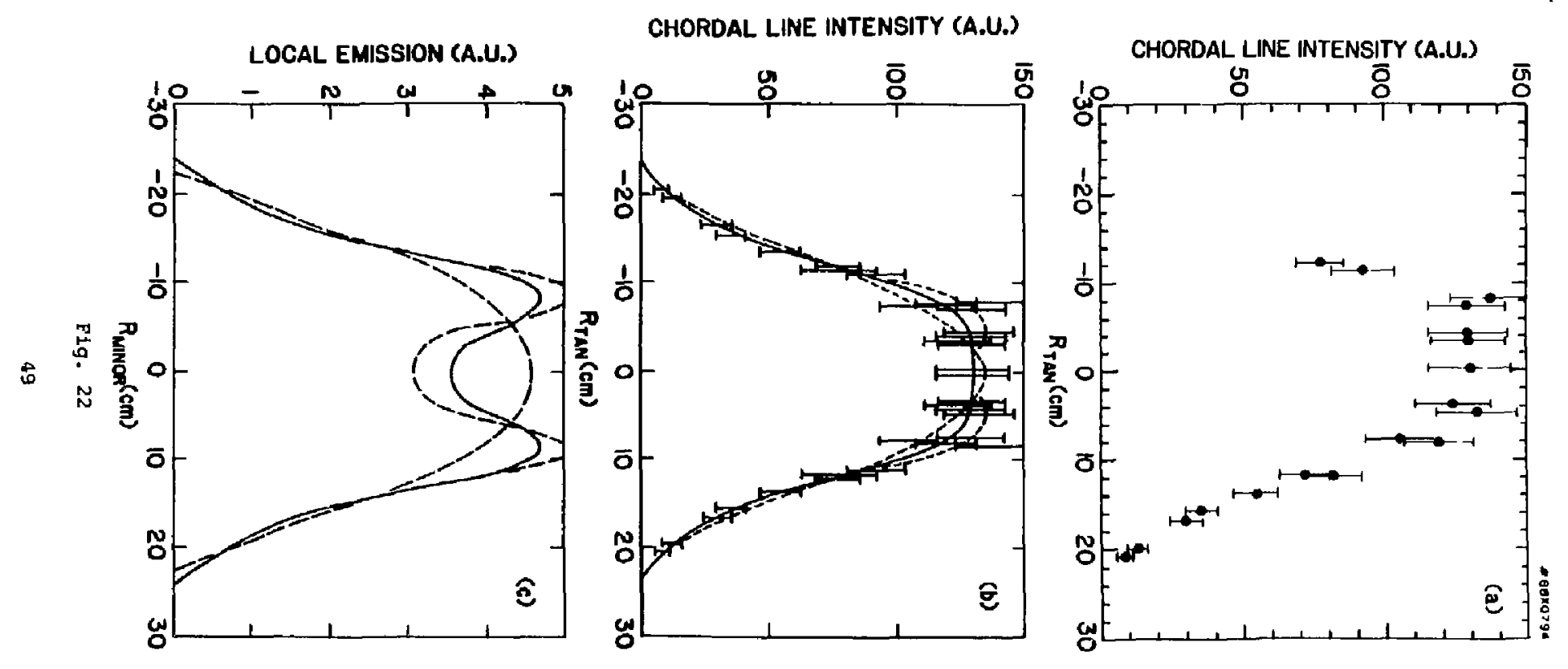



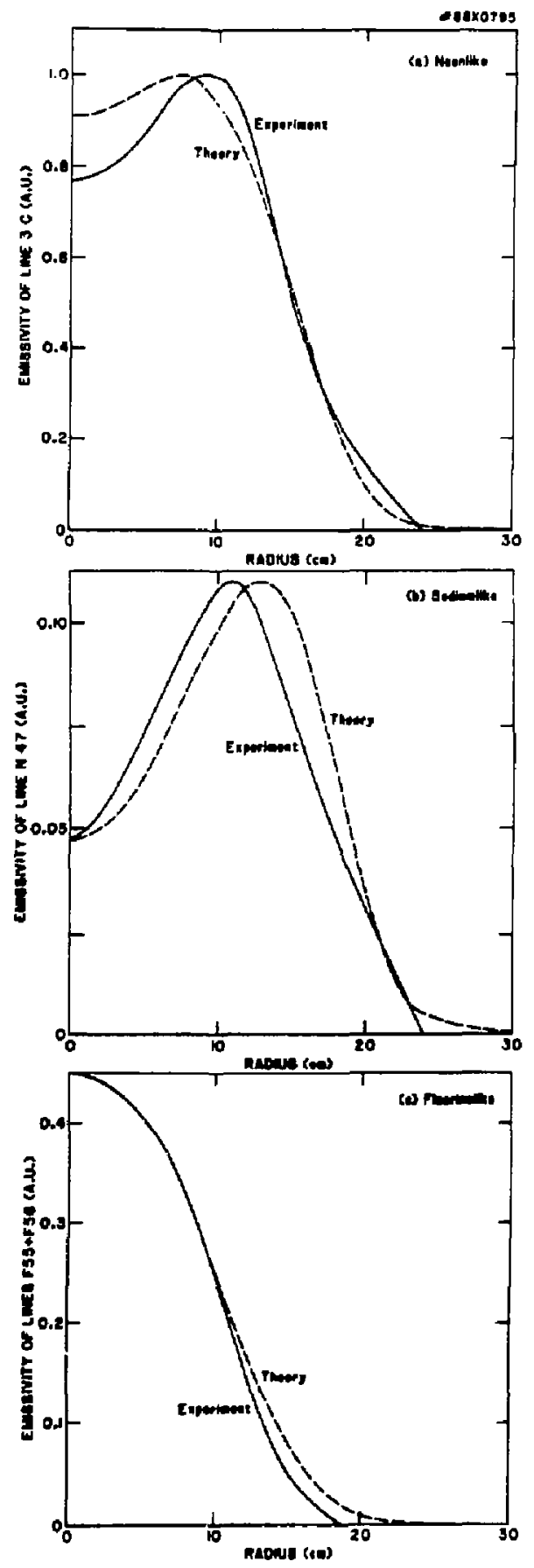

Fig. 23 